This article was downloaded by:[Swets Content Distribution]

On: 26 July 2007

Access Details: [subscription number 768307933]

Publisher: Psychology Press

Informa Ltd Registered in England and Wales Registered Number: 1072954

Registered office: Mortimer House, 37-41 Mortimer Street, London W1T 3JH, UK

\title{
APHASIOLOGY Aphasiology
}

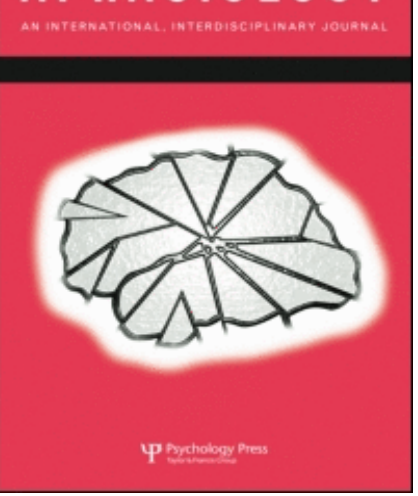

Publication details, including instructions for authors and subscription information: http://www.informaworld.com/smpp/title content=t713393920

The impact of phonological or semantic impairment on delayed auditory repetition: Evidence from stroke aphasia and semantic dementia

Elizabeth Jefferies ${ }^{\text {a. }}$ Jenni Crisp ${ }^{\text {b }}$; Matthew A. Lambon Ralph ${ }^{a}$

a University of Manchester, UK

b North Tyneside Primary Care Trust and University of Newcastle, UK

Online Publication Date: 01 September 2006

To cite this Article: Jefferies, Elizabeth, Crisp, Jenni and Ralph, Matthew A. Lambon (2006) 'The impact of phonological or semantic impairment on delayed auditory

repetition: Evidence from stroke aphasia and semantic dementia', Aphasiology, 20:9, $963-992$

To link to this article: DOI: $10.1080 / 02687030600739398$

URL: http://dx.doi.org/10.1080/02687030600739398

\section{PLEASE SCROLL DOWN FOR ARTICLE}

\section{Full terms and conditions of use: http://www.informaworld.com/terms-and-conditions-of-access.pdf}

This article maybe used for research, teaching and private study purposes. Any substantial or systematic reproduction, re-distribution, re-selling, loan or sub-licensing, systematic supply or distribution in any form to anyone is expressly forbidden.

The publisher does not give any warranty express or implied or make any representation that the contents will be complete or accurate or up to date. The accuracy of any instructions, formulae and drug doses should be independently verified with primary sources. The publisher shall not be liable for any loss, actions, claims, proceedings, demand or costs or damages whatsoever or howsoever caused arising directly or indirectly in connection with or arising out of the use of this material.

(c) Taylor and Francis 2007 


\title{
The impact of phonological or semantic impairment on delayed auditory repetition: Evidence from stroke aphasia and semantic dementia
}

\author{
Elizabeth Jefferies \\ University of Manchester, UK \\ Jenni Crisp \\ North Tyneside Primary Care Trust and University of Newcastle, UK \\ Matthew A. Lambon Ralph \\ University of Manchester, UK
}

\begin{abstract}
Background/Aims: This study aimed to evaluate the interactive account of repetition by examining the influence of factors that differentially tapped semantic and phonological processing in a case series of patients with semantic or phonological impairment.

Methods \& Procedures: We compared two patient groups: predominantly phonologically impaired cases with aphasia following cerebrovascular accident, and patients with semantic dementia. Immediate repetition was contrasted with repetition after a 5 -second filled delay, and lexicality, frequency, and imageability were manipulated-therefore both the task and the neuropsychological impairment biased processing in favour of either lexical-semantic or phonological capacities.

Outcomes \& Results: Substantial interactivity was observed between phonological/ semantic impairment and variables largely tapping these processes. The phonologically impaired patients showed substantial effects of lexicality and imageability that were larger in delayed than immediate repetition. The semantically impaired patients exhibited the complementary pattern, showing reduced effects of these lexical-semantic variables and a delay effect that was larger for more poorly comprehended, lowfrequency items. Semantic errors were related to phonological deficits whereas semantic impairment led to an increase in phonological errors. The phonologically impaired stroke cases also made more perseverative responses.

Conclusions: These findings support the view that repetition is underpinned by interaction between semantics and phonology within a single route and not by distinct lexical and sub-lexical pathways. The results also provide evidence of a continuum between phonological and deep dysphasia.
\end{abstract}

There are two opposing accounts of the way in which phonological and lexicalsemantic representations contribute to auditory repetition. According to dual-route accounts (Hanley \& Kay, 1997; Hanley, Kay, \& Edwards, 2002; Hillis \& Caramazza, 1991; McCarthy \& Warrington, 1984, 1987), there are distinct non-lexical and

Address correspondence to: Dr Elizabeth Jefferies, Neuroscience and Aphasia Research Unit, School of Psychological Sciences, University of Manchester, Oxford Road, Manchester, M13 9PL, UK. E-mail: beth.jefferies@manchester.ac.uk 
lexical-semantic pathways to repetition. The non-lexical route, which transforms auditory representations directly into an articulatory code without reference to word meaning, is critical for the repetition of nonwords. The lexical-semantic route utilises connections from input phonology to semantics and then to output phonology in order to support word repetition. In contrast, single-route models - for example, the interactive-activation (IA) model of Dell and colleagues (Dell \& O'Seaghda, 1992; Dell, Schwartz, Martin, Saffran, \& Gagnon, 1997; Foygel \& Dell, 2000) and the parallel distributed processing (PDP) framework of Plaut and Kello (1999) propose that interactivity between phonology and semantics underpins the repetition of all items. Within this single route, the repetition of nonwords is supported primarily by phonology, whereas lexical-semantic knowledge provides additional constraints on phonological activation for words.

Patients who have phonological deficits from either stroke or nonfluent progressive aphasia show poor repetition characterised by frequent phonological errors (Croot, Patterson, \& Hodges, 1998; Wilshire \& Fisher, 2004; Wilshire \& McCarthy, 1996). In contrast, individuals who show a profound loss of conceptual knowledge in the context of good phonology, e.g., patients with fluent progressive aphasia/semantic dementia (SD), have relatively intact repetition. This apparent dissociation speaks against the view that semantics plays a necessary role in the repetition of all items (e.g., the proposal of Plaut \& Kello, 1999). However, when the phonological system is stressed in delayed repetition or the immediate serial recall (ISR) of several items, SD patients show clearer effects of semantic status on repetition and make errors that resemble those made by phonologically impaired patients (Knott, Patterson, \& Hodges, 1997; see below). Interactivity between phonology and semantics may become more critical in repetition tasks that are highly demanding. The present study was motivated by the observation that repetition can look superficially similar in SD patients and phonologically impaired stroke aphasic patients when a delay is interposed between the stimulus and response: nevertheless, these groups should respond differently to variables that tap semantic and phonological processes if their difficulties in delayed repetition are underpinned by diverse impairments.

Several existing lines of evidence support the view that repetition is underpinned by interactivity between semantics and phonology within a single route. Semantically impaired patients, such as those with SD, show superior ISR for words that they still understand relatively well, compared with words that are more semantically degraded according to performance on semantic tests such as naming and wordpicture matching (Caza, Belleville, \& Gilbert, 2002; Forde \& Humphreys, 2002; Jefferies, Jones, Bateman, \& Lambon Ralph, 2004a, 2005; Jefferies, Patterson, Jones, Bateman, \& Lambon Ralph, 2004b; Knott et al., 1997; Knott, Patterson, \& Hodges, 2000; Patterson, Graham, \& Hodges, 1994). They make frequent phoneme migration errors when recalling word lists that they no longer fully understand, suggesting that semantic representations may help to constrain the order of phonemes in verbal short-term memory (Patterson et al., 1994). Similarly, normal participants make phoneme migration errors in ISR when lexical-semantic constraints are weak (Jefferies, Frankish, \& Lambon Ralph, 2006a; Treiman \& Danis, 1988).

Patients with a circumscribed semantic impairment also show poorer ISR for nonwords that are phonologically similar to semantically degraded words, compared with nonwords derived from better-understood words (Caza et al., 2002; Jefferies et al., 2005), suggesting a role for lexical-semantic representations in nonword as well 
as word repetition. This finding contradicts the strong form of the dual-route hypothesis which proposes that nonword repetition is achieved entirely through the non-lexical route. Lexical variables, such as the number of real-word neighbours, also have a demonstrable impact on nonword repetition in normal participants (Roodenrys \& Hinton, 2002).

Converging evidence for interactivity is provided by studies of stroke aphasia. Within this population, phonological and semantic impairment produce dissociable effects on repetition/ISR: poor semantic processing is associated with reduced effects of frequency and imageability, whereas poor phonological processing is associated with increased effects of these variables (N. Martin \& Saffran, 1997). In addition, patients can show specific difficulties in the retention of semantic or phonological information (R. Martin \& Lesch, 1996; R. Martin, Lesch, \& Bartha, 1999; R. Martin, Shelton, \& Yaffee, 1994).

A small number of aphasic patients with severe phonological deficits show a pattern termed "deep dysphasia"; that is, they make semantic errors in immediate single word repetition (e.g., camel $\rightarrow$ "horse"), show a large effect of semantic variables such as imageability in repetition, and are unable to repeat nonwords, suggesting that their repetition relies heavily on word meaning (for example, Howard \& Franklin, 1988; Katz \& Goodglass, 1990; Majerus, Lekeu, Van der Linden, \& Salmon, 2001; N. Martin \& Saffran, 1992; Michel \& Andreewsky, 1983; Valdois, Carbonnel, David, Rousset, \& Pellat, 1995). In accounting for the syndrome of deep dysphasia, the single-route account has the advantage that apparently disparate effects - such as the occurrence of both semantic and phonological errors and effects of lexicality and imageability in repetition - can be explained parsimoniously in terms of a single impairment. N. Martin, Saffran, and colleagues demonstrated that the key symptoms of deep dysphasia could be accounted for in Dell and O'Seaghda's (1992) model by postulating an abnormally fast decay of activation in all units (Martin, Dell, Saffran, \& Schwartz, 1994; Martin \& Saffran, 1992; Martin, Saffran, \& Dell, 1996). In a recent modification of this model, Foygel and Dell (2000) proposed that phonological and semantic processing could be impaired independently; consequently, deep dysphasia might arise from a specific impairment of phonological processing that renders repetition more reliant on lexical-semantic processing (see Wilshire \& Fisher, 2004). In contrast, two independent deficits might be postulated to explain deep dysphasia within the dual-route framework. The inability to repeat nonwords is suggestive of an impairment to the non-lexical route, whereas the large imageability effects and semantic errors shown by these patients are interpreted as resulting from additional weakness in the lexical-semantic route (see Hanley, Dell, Kay, \& Baron, 2004; Hanley \& Kay, 1997; Hanley et al., 2002).

The findings reviewed above are broadly consistent with Dell and colleagues' interactive-activation (IA) model (Dell \& O’Seaghda, 1992; Dell et al., 1997), which incorporates three distinct processing levels: phonological, lexical, and semantic. Each level consists of localist nodes, linked by bidirectional connections to the nodes in the adjacent layers. Activation spreads forwards and backwards between the levels during every processing cycle so that in repetition, reverberating input from the lexical and semantic layers helps to sustain rapidly decaying phonological activation. The PDP framework (e.g., Patterson et al., 1994; Plaut \& Kello, 1999) makes similar predictions but employs a rather different architecture. This approach posits distinct semantic and phonological representations but not a separate lexical level. The phonological system develops pattern completion properties for familiar items by 
virtue of the fact that the phonological elements of words are always produced together. A second source of constraint is provided by the semantic system. As semantic activation co-occurs with phonology during word comprehension and production, input from semantic memory helps to bolster the correct phonological pattern and suppresses activation of erroneous phonology. Both of these models predict that semantic impairment will increase phonological errors in repetition.

This study aims to evaluate the interactive account of repetition by examining the way in which factors that differentially tap semantic processing (e.g., lexicality and imageability) and phonological processing (e.g., the presence of a delay prior to repetition) interact with semantic or phonological impairment in SD and stroke aphasia respectively. Patients with phonological impairment should perform poorly when a brief delay is imposed between the stimulus and response because phonological activation is thought to decay rapidly and phonological impairment will therefore be exacerbated by a delay. These patients are also expected to have particular difficulty repeating stimuli such as nonwords, which derive little support from lexical-semantic representations and thus rely more heavily on phonology. Similarly, phonologically impaired patients should show increased effects of semantic variables such as imageability. Highly imageable words have been assumed by a number of authors to have "richer" semantic representations than less imageable words, perhaps because they are associated with a larger number of semantic features (Jones, 1985; Plaut \& Shallice, 1991). Consequently, when repetition is forced to rely heavily on lexical-semantic processing due to phonological impairment, items that are well supported by such processing (i.e., highly imageable words) are repeated with a higher degree of accuracy than items that are not well supported (i.e., lower-imageability words). Larger than normal imageability effects emerge as a direct consequence of phonological impairment according to this interactive framework, rather than from additional damage somewhere along the lexical-semantic route (see Martin \& Saffran, 1997). The interactive perspective also predicts that semantic effects should be greater following a delay: this biases the system towards greater reliance on semantic processing and will have a more catastrophic impact on the repetition of stimuli that derive little support from lexical-semantic activation, such as nonwords. Finally, the interactive framework anticipates that phonological impairment should increase the likelihood of both phonological and semantic errors in repetition, in line with the performance of deep dysphasic patients (see Martin \& Saffran, 1992): phonological errors reflect difficulty in maintaining a veridical phonological representation, and semantic errors reflect an increased reliance on lexical-semantic processing.

In contrast, semantically impaired patients might be expected to show a reduction in the impact of word meaning on repetition. Lexicality effects should be smaller in this patient group, as lexical-semantic support for words over nonwords will be eroded. Previous studies have demonstrated that semantic deficits are also associated with reduced effects of word frequency and imageability in repetition (Martin \& Saffran, 1997). However, patients with semantic dementia show degradation of semantic knowledge that is highly sensitive to word frequency. The meaning of lower-frequency words/concepts typically degrades earlier in the condition (Funnell, 1995), so high-frequency words may receive a stronger boost from lexical-semantic representations relative to lower-frequency words. This should increase, rather than decrease, the impact of word frequency on repetition in SD. In addition, word frequency/degree of semantic degradation should interact with delay - in immediate 
repetition, the good phonology of SD patients might enable them to retain even poorly understood words. However, after a delay, the phonological trace is no longer adequate for accurate repetition, and this should be more apparent for lowerfrequency words that are more semantically degraded. It is more difficult to know what to predict for imageability. If highly imageable words have more semantic features (Jones, 1985; Plaut \& Shallice, 1991), then they might be expected to be relatively resistant to semantic degradation. If so, SD patients should show enhanced effects of imageability as well as frequency in repetition. On the other hand, given that imageability effects are thought to reflect the normal operation of the semantic system, they might be reduced in size in SD. Finally, semantic impairment is expected to increase the occurrence of phonological errors in repetition due to a lessening of semantic constraints that reinforce the correct phonological pattern. These predictions for phonologically and semantically impaired patients are summarised in Table 1.

In this work we investigated the impact of phonological or semantic impairment on the repetition of a group of $12 \mathrm{CVA}$ and $6 \mathrm{SD}$ patients. SD and CVA patients have previously been studied in order to assess the contribution of phonological and semantic processes in repetition, but they have not been directly compared on the same tasks. We present analyses that (1) compare these two groups directly and (2) collapse across the groups to examine the influence of phonological and semantic skills on repetition. Previous investigations of the role of phonological and semantic processes in repetition (reviewed above) have relied largely on descriptions of single cases (although see N. Martin \& Saffran, 1997, for a notable exception). The advantage of the case-series approach adopted here is that it is possible to investigate how factors tapping semantic/phonological processing vary systematically as a function of semantic/phonological impairment. For example, does the size of the imageability effect vary with the degree of phonological impairment (as proposed by Martin \& Saffran, 1997) or with lexical-semantic damage (as proposed by Hanley \& Kay, 1997; Hanley et al., 2002)? Is there a continuum between phonological and deep dysphasia explicable in terms of the severity of phonological impairment? To what extent does repetition reflect interactivity between semantics and phonology, in line with the predictions in Table 1 ?

We examined both immediate repetition and delayed repetition following articulatory suppression because theoretical considerations (see above) and empirical

TABLE 1

Predicted impact of semantic and phonological impairment on repetition according to interactive models

\begin{tabular}{|c|c|c|}
\hline & Semantic impairment (semantic dementia) & Phonological impairment \\
\hline $\begin{array}{l}\text { Delay before } \\
\text { response }\end{array}$ & $\begin{array}{l}\text { Small effect; biggest for poorly comprehended } \\
\text { words }\end{array}$ & Large effect; biggest for nonwords \\
\hline Lexicality & Reduced effect & Increased effect \\
\hline Imageability & Unclear & Increased effect \\
\hline Frequency & $\begin{array}{l}\text { Increased effect reflecting degree of semantic } \\
\text { degradation }\end{array}$ & Increased effect \\
\hline $\begin{array}{l}\text { Phonological } \\
\text { errors }\end{array}$ & Increased in frequency & Increased in frequency \\
\hline Semantic errors & No semantic errors & Increased in frequency \\
\hline
\end{tabular}


findings (Jefferies et al., 2006a) suggest that lexical-semantic factors may play a greater role in repetition tasks when phonological maintenance is especially challenging. In delayed repetition, phonological output is not tightly constrained by incoming phonology; consequently long-term knowledge of the sounds and meanings of familiar words should make a larger contribution to phonological output. In line with this suggestion, phonological errors have been observed previously in delayed but not immediate repetition for a few SD patients (Knott et al., 1997, 2000): this study explores the generality of these findings in both SD and CVA. The study therefore presents an opportunity to establish if N. Martin and Saffran's (1997) findings for two-word lists generalise to a new task (delayed repetition) and a new patient population (SD).

\section{METHOD}

\section{Participants}

A total of 12 phonologically impaired CVA patients (PI-CVA) and 6 semantic dementia (SD) patients took part in the study (see Table 2 for background information). These groups had contrasting deficits: the PI-CVA group had more intact semantic memory but poorer phonological processing, whereas the SD patients had severe semantic impairment but relatively good phonology (see Tables 2 and 3). These groups were compared in order to explore the impact of semantic and phonological impairment on repetition. There was, however, some overlap between the semantic/phonological abilities of the patients in the two groups: some of the CVA patients had semantic as well as phonological difficulties. We therefore also examined associations between repetition and semantic/phonological deficits collapsing across the two groups (following the methods of N. Martin \& Saffran, 1997).

Semantic dementia. SD is the temporal variant of frontal-temporal dementia and is associated with progressive bilateral focal atrophy of the anterior infero-temporal neocortex, which results in a specific and progressive impairment of semantic memory (Hodges, Patterson, Oxbury, \& Funnell, 1992; Snowden, Goulding, \& Neary, 1989). Patients with SD display a highly uniform pattern of impairments: they are anomic in spontaneous speech and picture naming and have impaired comprehension on both verbal and non-verbal tasks. In contrast, their perceptual and spatial skills, new episodic learning, non-verbal reasoning, syntax, and phonology remain largely intact (Hodges et al., 1992; Snowden, Neary, \& Mann, 1996). SD patients have fluent speech that is largely free from phonological errors. Likewise, their single word repetition is excellent and they typically have good digit span (Jefferies et al., 2004b). They are able to discriminate between pairs of words that differ by a single phonetic feature (such as hat/cat) and can perform phoneme addition/subtraction (e.g., "shale" $\rightarrow$ ALE), at least until the late stages of the disease (Jefferies et al., 2005). Even late-stage patients continue to be sensitive to the effects of phonological similarity in ISR (Jefferies, Patterson, Bateman, Jones, \& Lambon Ralph, 2006b). These findings support the characterisation of SD as semantic impairment in the context of good phonology. SD patients do show poor repetition of items that they no longer fully understand, especially in demanding tasks such as delayed repetition or ISR (see Jefferies et al., 2004a, for a review), 


\begin{tabular}{|c|c|c|c|c|c|c|c|c|c|c|c|c|c|c|c|c|c|c|c|c|c|c|c|}
\hline & 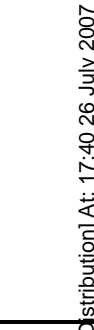 & & & & ackg & rounc & info & rmati & $\begin{array}{r}\mathrm{T} \\
\text { on an }\end{array}$ & $\begin{array}{l}\text { ABLE } \\
\text { d sen }\end{array}$ & $\begin{array}{l}2 \\
\text { nanti }\end{array}$ & c skills & s for & each & patier & & & & & & & & \\
\hline \multirow[b]{2}{*}{ Name } & 离 & & & & & & $P I-C$ & & & & & & & & & & $D$ & & & & ean & Cont & rols \\
\hline & 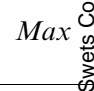 & $L R$ & $M M$ & $R J$ & $R S$ & $A B$ & $N S$ & $M R$ & $B N$ & $T J$ & $P G$ & $T H$ & $D B$ & $S J$ & $E K$ & $K I$ & $J T$ & GT & $M K$ & $\begin{array}{c}P I- \\
C V A \\
\end{array}$ & $S D$ & Mean & $\begin{array}{l}\text { Cut }- \\
\text { off } t\end{array}$ \\
\hline Age & & 58 & 58 & 40 & 64 & 83 & 51 & 72 & 52 & 60 & 66 & 48 & 61 & 60 & 60 & 65 & 66 & 71 & 68 & 59 & 65 & & \\
\hline Years post-onset & & $\begin{array}{ll}D_{0}^{2} & 13 \\
D_{0} & 13\end{array}$ & 10 & 2 & 3 & 1 & 8 & 2 & 4 & 6 & 2 & 3 & 1 & 3 & 5 & 4 & 4 & 5 & 3 & 4.6 & 4.0 & & \\
\hline $\begin{array}{l}\text { Spoken word-picture } \\
\text { match }\end{array}$ & 30 올 & 29 & 29 & 29 & 21 & 30 & 29 & 29 & 25 & 28 & 30 & 30 & 30 & 28 & 23 & 19 & 18 & 11 & 5 & 28.3 & 17.3 & $\$$ & 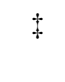 \\
\hline $\begin{array}{l}\text { Written word-picture } \\
\text { match }\end{array}$ & 30 & 27 & 28 & 29 & 29 & 29 & 29 & 30 & 24 & 29 & 30 & 29 & 30 & NT & NT & NT & NT & NT & NT & 28.6 & NT & 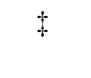 & 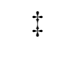 \\
\hline Picture association & 30 & 20 & 22 & 23 & 26 & 24 & 22 & 22 & 15 & 17 & 27 & 28 & 25 & 21 & 13 & 8 & 16 & 15 & 9 & 22.6 & 13.7 & 27.6 & 24.8 \\
\hline $\begin{array}{l}\text { Picture naming } \\
\text { Graded synonyms }\end{array}$ & 30 & 12 & 15 & 15 & 15 & 12 & 21 & 22 & 18 & 25 & 25 & 29 & 29 & 13 & 8 & 8 & 4 & 4 & 1 & 19.8 & 6.3 & $\star$ & $\ddagger$ \\
\hline Auditory: concrete & 25 & 15 & 14 & 14 & 8 & 18 & 18 & 14 & 10 & 17 & 23 & 22 & 14 & 16 & 14 & NT & NT & 14 & NT & 15.6 & 14.7 & 21.0 & 15.0 \\
\hline Written: concrete & 25 & 9 & 19 & 13 & 18 & 23 & 19 & 11 & 16 & 18 & 22 & 24 & 15 & NT & NT & NT & NT & NT & NT & 17.3 & NT & 23.9 & 21.4 \\
\hline Auditory: abstract & 25 & 15 & 13 & 14 & 10 & 19 & 18 & 14 & 13 & 19 & 19 & 21 & 16 & 12 & 12 & NT & NT & 9 & NT & 15.9 & 11.0 & 21.0 & 14.0 \\
\hline Written: abstract & 25 & 16 & 11 & 18 & 11 & 20 & 15 & 15 & 13 & 20 & 24 & 19 & 15 & NT & NT & NT & NT & NT & NT & 16.4 & NT & 23.3 & 20.0 \\
\hline $\begin{array}{l}\text { Frequency by imageability } \\
\text { synonyms }\end{array}$ & 96 & 46 & 51 & 68 & 54 & 74 & 80 & 60 & 68 & 72 & 85 & 86 & 77 & 78 & 68 & 58 & NT & 48 & 30 & 68.4 & 56.2 & 94.5 & 91.0 \\
\hline
\end{tabular}

PI-CVA= phonologically impaired cerebrovascular accident patient. $\mathrm{SD}=$ semantic dementia. $\mathrm{NT}=$ not tested. ${ }^{\dagger}=$ cut-off for normal performance at $2 S D$ below the mean. $\sharp=$ controls expected to be at ceiling on these easy tests. Picture association test=Shortened Camel and Cactus test used by Bozeat et al., 2000. Graded synonyms test by Warrington et al., 1998. Details of each test are given in the Method section. 


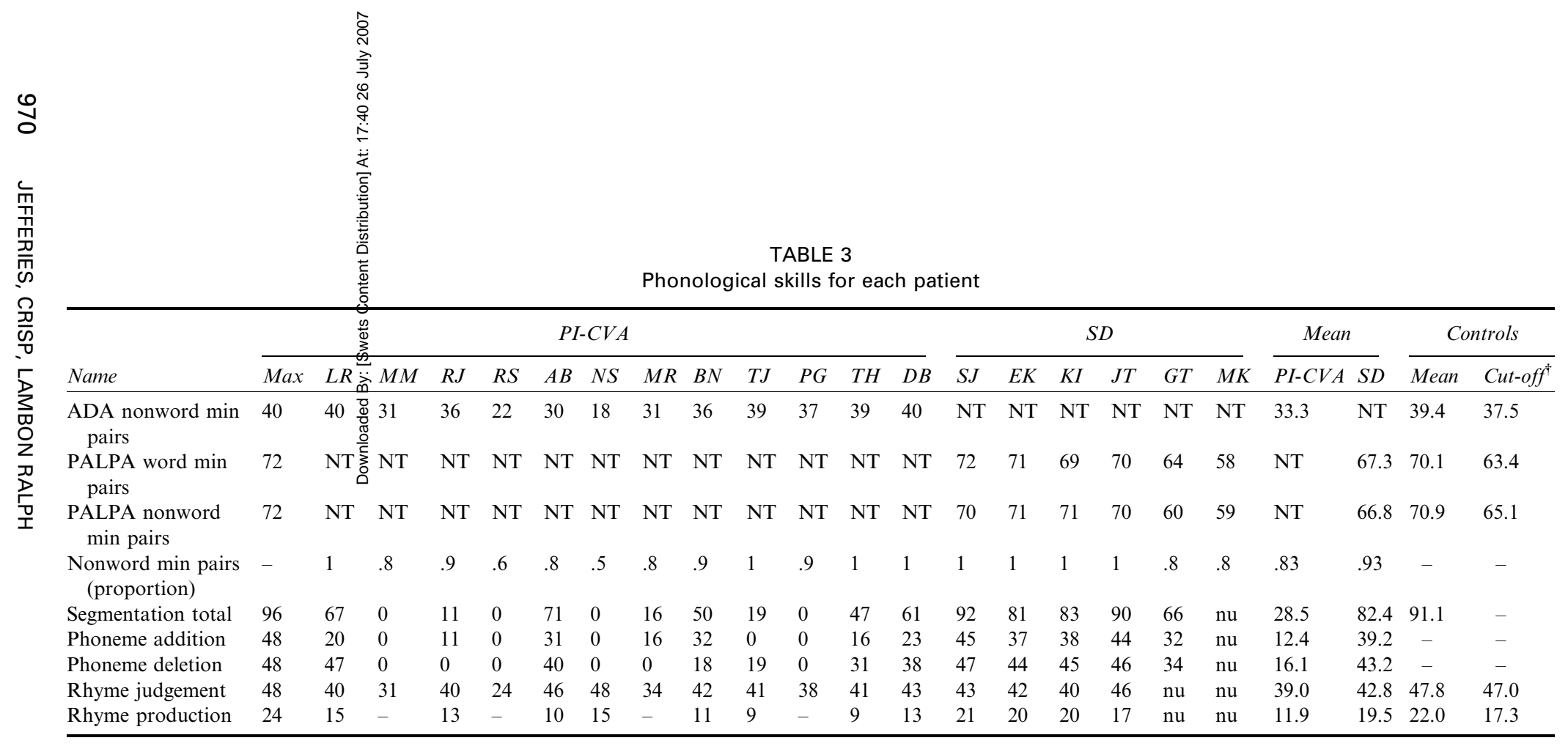

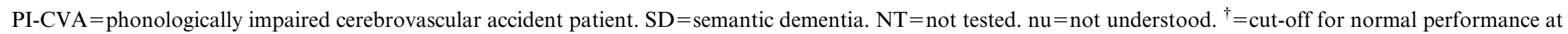
$2 S D$ below the mean. Min pairs=minimal pairs. ADA=Action for dysphasic adults (Franklin et al., 1992). PALPA=Psycholinguistic Assessments of Language Processing in Aphasia (Kay et al., 1992). Details of each test are given in the Method section. 
indicating that semantic memory makes a crucial contribution to phonological stability/maintenance. It should also be noted that as the disease progresses, patients show increasing difficulty on tasks such as phoneme segmentation and nonword repetition/recall. Although this pattern is consistent with the notion that additional phonological problems develop in late-stage SD, these tasks are not purely phonological and are demonstrably sensitive to semantic degradation. For example, nonwords that are phonologically similar to relatively well-understood words are recalled more accurately than nonwords that are derived from more semantically degraded words (Jefferies et al., 2005). Therefore, late-stage SD patients' difficulties on "phonological" tasks, which are observable in some of the patients examined here, may result from their severe semantic deficits.

Phonologically impaired cerebrovascular accident (PI-CVA) group. The PI-CVA group were recruited for a study on phonological and deep dyslexia (Crisp \& Lambon Ralph, 2006) and were therefore selected according to their reading performance, rather than their phonological skills. However, neuropsychological tests revealed that all the PI-CVA patients had moderate to severe phonological impairment (see Table 3). A subset of this group also showed deficits on tests of semantic memory although these problems were milder than those shown by the SD cases (see Table 2). This group can therefore be characterised as phonologically impaired but relatively semantically intact. Both fluent and non-fluent speakers were included. None of the patients had marked apraxia of speech (although subtle difficulties of this nature were possibly present in case RS). Further details of these patients are provided by Crisp and Lambon Ralph (2006).

\section{Phonological and semantic abilities in the two groups}

We examined the performance of the two patient groups on a battery of semantic and phonological tests to allow consideration of the impact of semantic/ phonological impairment on repetition. Four semantic tests were used. (1) Auditory word-picture matching: this test required patients to select the picture named aloud by the experimenter. There were 30 targets, each presented with 9 semantically related foils. The PI-CVA patients were also asked to perform a written version of this task. Their performance was largely comparable across the two versions, although one patient (RS), with auditory input problems/word deafness, was more accurate for written than spoken word-picture matching. ${ }^{1}$ (2) The Camel and Cactus test of semantic association (Bozeat, Lambon Ralph, Patterson, Garrard, \& Hodges, 2000). This was similar to the Pyramids and Palm Trees test (Howard \& Patterson, 1992) and used the same 30 items as the word-picture matching test described above. Patients were asked to decide which of four pictures was most closely related in meaning to a target picture (e.g., camel: tree, sunflower, cactus, rose). The four choices were drawn from the same semantic category. (3) Patients were asked to name the same 30 targets from black and white line drawings. (4) Imageability by frequency synonym judgement test. Participants were asked to choose which of three written words was most similar in meaning to a written target (e.g., keep: become, save, put). There were 96 trials that orthogonally varied

\footnotetext{
${ }^{1}$ Despite this difference, the PI-CVA patients showed better performance than the SD patients on the auditory version of the task.
} 
imageability (low, medium, high) and frequency (low and high). All four words within a trial were matched for these two factors such that the trials as a whole varied consistently on these two variables. The words were read aloud to the SD patients during the test because of their marked surface dyslexia. As the words were not also read aloud to the PI-CVA patients, the test is unlikely to be a pure measure of the comprehension of this reading-impaired group. However, the PI-CVA patients also completed the graded synonyms test (Warrington, McKenna, \& Orpwood, 1998) using auditory and written presentation and the majority of patients showed little difference between the two modalities.

There were also four phonological tests. (1) Phoneme manipulation (Patterson \& Marcel, 1992). There were two versions of this task. In the phoneme subtraction version, the patients were asked to delete the first sound of an item and say what remained (e.g., vale $\rightarrow$ ale). In the phoneme addition version, patients were asked to add a phoneme to the rhyme of an item (e.g., ale $\rightarrow$ vale). All of the items were monosyllabic and the same 48 items were used in the two versions of the task. The lexicality of the stimulus and the target response was manipulated. There were four conditions: word $\rightarrow$ word; word $\rightarrow$ nonword; nonword $\rightarrow$ word; nonword $\rightarrow$ nonword. (2) Rhyme judgement (Patterson \& Marcel, 1992): This task required patients to judge whether or not two spoken words rhymed (e.g., white-kite). There were 48 trials. Half of the 24 non-rhyming trials were composed of two phonologically similar words (e.g., tick-tin). (3) Rhyme production (Patterson \& Marcel, 1992): Patients were asked to produce a word that rhymed with 24 spoken words. (4) Minimal pairs: Patients made same/different judgements for pairs of auditorily presented nonwords that differed by a single phonetic feature (e.g., mivniv). The available data for the two patient groups on this task are from two comparable tests: the PI-CVA patients were tested on the nonword minimal pairs test from the ADA battery (Franklin, Turner, \& Ellis, 1992), whereas the SD patients were tested on the minimal pairs test from the PALPA (Kay, Lesser, \& Coltheart, 1992). While it is obviously less than ideal that the patients were tested on different sets of items, these data still provide a guide to the auditory discrimination abilities of the two groups.

Several of the PI-CVA patients were unable to perform the phoneme manipulation and rhyme production tasks. For these patients, testing was discontinued and a score of zero was assumed. There are also missing data on the phoneme manipulation and rhyme tests for two of the later-stage SD patients. These cases were unable to comprehend the task instructions (for example, they did not understand the notion of "rhyme"). These values are treated as missing below. ${ }^{2}$

Across these tests, the SD patients were semantically impaired yet relatively phonologically intact, whereas the PI-CVA patients were phonologically impaired but more semantically intact (see Tables 2 and 3). The SD patients showed greater impairment than the PI-CVA group across a range of semantic tests: spoken wordpicture matching, $t(16)=4.26, p=.001$; semantic association, $t(16)=4.26, p=.001$; and picture naming, $t(16)=4.77, p=.0002$. However, they did not differ significantly on the synonym judgement test, $t(15)=1.55, n s$, possibly because the PI-CVA

\footnotetext{
${ }^{2}$ Nevertheless, the patient groups were significantly different on these tasks even when the zeros obtained by the PI-CVA patients were treated as missing values.
} 
patients' poor reading (their phonological/deep dyslexia) contributed to their difficulties on this task. The PI-CVA patients performed more poorly than the SD patients on two measures of phonological skill: phoneme manipulation, $t(15)=4.05$, $p=.001$, and rhyme production, $t(14)=3.63, p=.003$. However, the two groups did not differ significantly on two other measures: rhyme judgement $t(14)=1.09, n s$, and minimal pair discrimination $t(16)=1.25, n s$.

Collapsing across groups, there were strong correlations between the four semantic tasks (word-picture matching and semantic association: $r=.72, p=.001$; word-picture matching and naming: $r=.77, p=.0002$; naming and semantic association: $r=.71, p=.001$; word-picture matching and synonym judgement: $r=.73, p=.001$; naming and synonym judgement: $r=.74, p=.001$; semantic association and synonym judgement: $r=.58, p=.02$ ). There were also marked correlations between the phonological tasks. Phoneme manipulation correlated with all three of the other phonological tasks (rhyme judgement: $r=.53, p=.04$; rhyme production: $r=.78, p=.0004$; minimal pairs: $r=.59, p=.01$ ). Rhyme production also correlated with rhyme judgement $(r=.71, p=.002)$.

Word-picture matching, semantic association, and naming did not positively correlate with any of the phonological measures. Similarly, phoneme segmentation, rhyme production, and minimal pair discrimination did not positively correlate with any of the semantic measures. ${ }^{3}$ However, there was a positive correlation between synonym judgement and rhyme judgement $(r=.58, p=.02)$, possibly because both tasks tapped working memory and required decision making.

An overall estimate of each participant's semantic and phonological performance was obtained using factor analysis (as in Lambon Ralph, Moriarty, \& Sage, 2002). For semantics, we used the three semantic tasks for which data were available for every patient (word-picture matching, naming, semantic association). The singlefactor solution accounted for $82 \%$ of the variance. For phonology, we used the two phonological tasks for which there were least missing data (phoneme manipulation and minimal pair discrimination). ${ }^{4}$ Again, a single-factor solution was derived that accounted for $79 \%$ of the variance. We did not obtain a composite measure of phonology for one SD patient, MK, due to missing data. The factor scores from these analyses were used as a measure of each patient's semantic/phonological impairment in subsequent analysis.

\section{Repetition tasks}

In each of three tasks, immediate and delayed repetition was assessed in a single trial. The experimenter presented the items auditorily. Participants attempted to repeat

\footnotetext{
${ }^{3}$ There were some negative correlations between phonological and semantic measures, presumably reflecting the fact that the semantically impaired SD patients had relatively good phonology whereas the phonologically impaired CVA patients had relatively good semantics.

${ }^{4}$ Several of the stroke aphasic patients were unable to complete the segmentation task, presumably due to their severe phonological difficulties, and were assigned a score of zero which was included in the factor analysis. We also computed a phonological factor score using minimal pair discrimination and rhyme judgement; for these tasks, all of the stroke patients were off floor. The two factor scores were very highly correlated $(r=.85 ; p<.0001)$. Both significantly correlated with the size of the imageability effect in delayed repetition although the alternative phonological factor score did not correlate with the effect of delay in repetition.
} 
each one both immediately and again after a 5-second delay. The delay was filled with phonological production (see below). No feedback was given about the accuracy of responses.

(1) The first task involved 96 words and crossed two levels of word frequency with three levels of imageability (the targets from the imageability by frequency synonym judgment test; referred to as Word Set 1 below). As the highimageability words in Set 1 were significantly shorter than the low-imageability words, a matched subset of 72 items was selected. In this subset, syllable and phoneme length were held constant across the different levels of frequency and imageability. The characteristics of these words are shown in the Appendix. For the PI-CVA patients, the delay between the immediate and second repetition was filled with rehearsal of their own name. ${ }^{5}$ The SD group was asked to count aloud from 1 during the delay.

(2) Both groups of participants were asked to count aloud from 1 during the 5second delay for Word Set 2, making it possible to see if the results from the first word set would hold for a new set of items when the two patient groups performed identical tasks. There was some variation in the rate of participants' counting, with the SD patients typically counting faster than the PI-CVA group. This word set also crossed frequency with imageability and contained two levels of each variable. The 56 items considered here comprised a matched subset of those tested (62 for the SD patients and 120 for the PI-CVA patients). The highand low-frequency/imageability words were equivalent in terms of phoneme and syllable length (see Appendix).

(3) The third task involved nonword repetition. The 48 nonwords were derived from the low-frequency words in Word Set 1 and were matched to them for syllable length and number of phonological neighbours. Other methodological details were as described for Word Set 1.

\section{RESULTS}

\section{Accuracy: Difference between patient groups}

As the results were very similar for the complete word sets and the matched subsets in which length was held constant across the levels of frequency/imageability, only the matched subsets are considered below. Immediate self-corrections were counted as correct in these analyses.

Words: Set 1. Immediate and delayed repetition data were obtained for 11 PICVA patients and 6 SD patients. One PI-CVA patient was unable to complete the delayed repetition task and was excluded from the analysis. The results for each group are shown in Figure 1 and individual patient data are provided in Table 4. The influence of delay, frequency, and imageability on repetition in the two patient groups was compared by subjects $\left(F_{1}\right)$ and by items $\left(F_{2}\right)$ using analysis of variance (ANOVA). Delay had a greater impact on the repetition of the PI-CVA patients, in line with their more severe phonological impairments, $F_{l}(1,15)=7.6, p=.02 ; F_{2}(1$,

\footnotetext{
${ }^{5}$ Pilot work had identified this as the most reliable method of getting this group with sometimes severe expressive aphasia to fill the delay with overt articulatory suppression.
} 


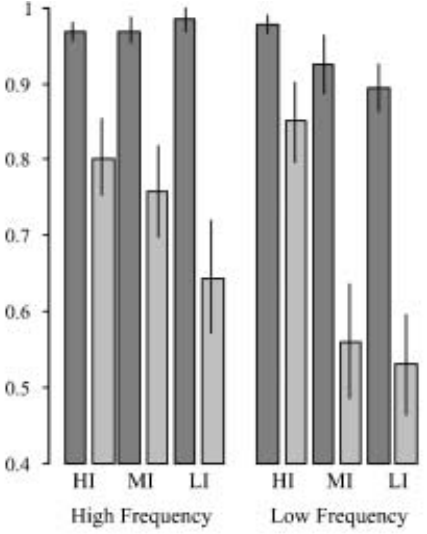

Phonologically impaired CVA
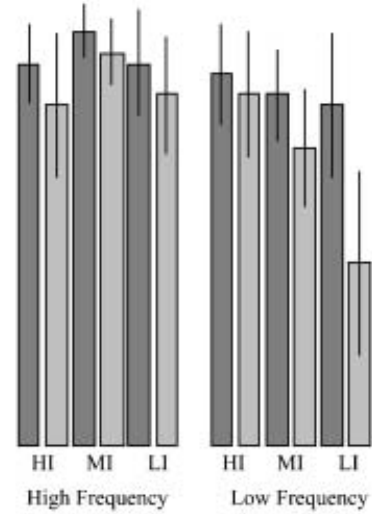

$\square$ Immediate repetition

$\square$ Delayed repetition

Note: Each set of bars shows immediate and delayed repetition for one level of frequency (high/low) in one group of patients at three levels of

Figure 1. The influence of frequency and imageability on immediate and delayed repetition.

$66)=70.8, p<.0001$. Immediate repetition was superior to delayed repetition for both the PI-CVA patients, $F_{l}(1,10)=29.2, p=.001 ; F_{2}(1,66)=231.2, p<.0001$, and the SD patients, $F_{1}(1,5)=7.6, p=.04 ; F_{2}(1,66)=28.0, p<.0001$.

High-frequency words were repeated more accurately than low-frequency words by both the SD patients, $F_{1}(1,5)=12.03, p=.02 ; F_{2}(1,66)=7.3, p=.01$, and the PICVA patients, $F_{l}(1,10)=20.1, p=.001 ; F_{2}(1,66)=10.0, p=.002$. Frequency had an equivalent effect on the two groups, $F_{1}(1,15)<1 ; F_{2}(1,66)<1$. For the SD patients, the effect of the delay was modulated by frequency, $F_{l}(1,5)=11.0, p=.02$; $F_{2}(1,66)=4.9, p=.03$ : delay had relatively little impact on the high-frequency, more semantically intact items, Bonferroni $t_{1}(5)=1.3, n s ; t_{2}(35)=3.0, p=.01$, but a more substantial effect on the poorly comprehended/low-frequency items, Bonferroni $t_{1}(5)=4.3, p=.01 ; t_{2}(35)=3.9, p=.0008$. In contrast, delay did not interact with frequency for the PI-CVA group, $F_{l}(1,10)=3.8, p=.08 ; F_{2}(1,66)=1.7$, ns. The three-way interaction between patient group, frequency and delay did not reach significance, $F_{1}(1,15)<1 ; F_{2}(1,66)<1$.

High-imageability words were repeated more accurately than low-imageability words by the PI-CVA patients, $F_{l}(2,20)=13.7, p=.0002 ; F_{2}(2,66)=15.2$, $p<.0001$. In contrast, the SD patients did not show a significant main effect of imageability, $F_{l}(2,10)=1.8, n s ; F_{2}(2,66)=2.0, n s$. Imageability affected the repetition of the PI-CVA patients more strongly than the $\mathrm{SD}$ patients, $F_{l}(2,30)=$ $2.5, p=.1 ; F_{2}(2,66)=3.6, p=.03$. In both groups, the delay had a greater influence on words that were lower in imageability, PI-CVA: $F_{l}(2,20)=11.4, p=.0005$; $F_{2}(2,66)=12.2, p<.0001 ; \mathrm{SD}: F_{1}(2,10)=7.5, p=.01 ; F_{2}(2,66)=3.9, p=.03$. This interaction between delay and imageability was larger for the PI-CVA patients, $F_{1}(2,30)=2.8, p=.08 ; F_{2}(2,66)=3.5, p=.04$.

There was an interaction between frequency and imageability, $F_{l}(2,30)=7.1$, $p=.003 ; F_{2}(2,66)=4.5, p=.02$, which did not vary significantly with patient group, $F_{1}(2,30)<1 ; F_{2}(2,66)<1$. The size of the frequency by imageability interaction was 


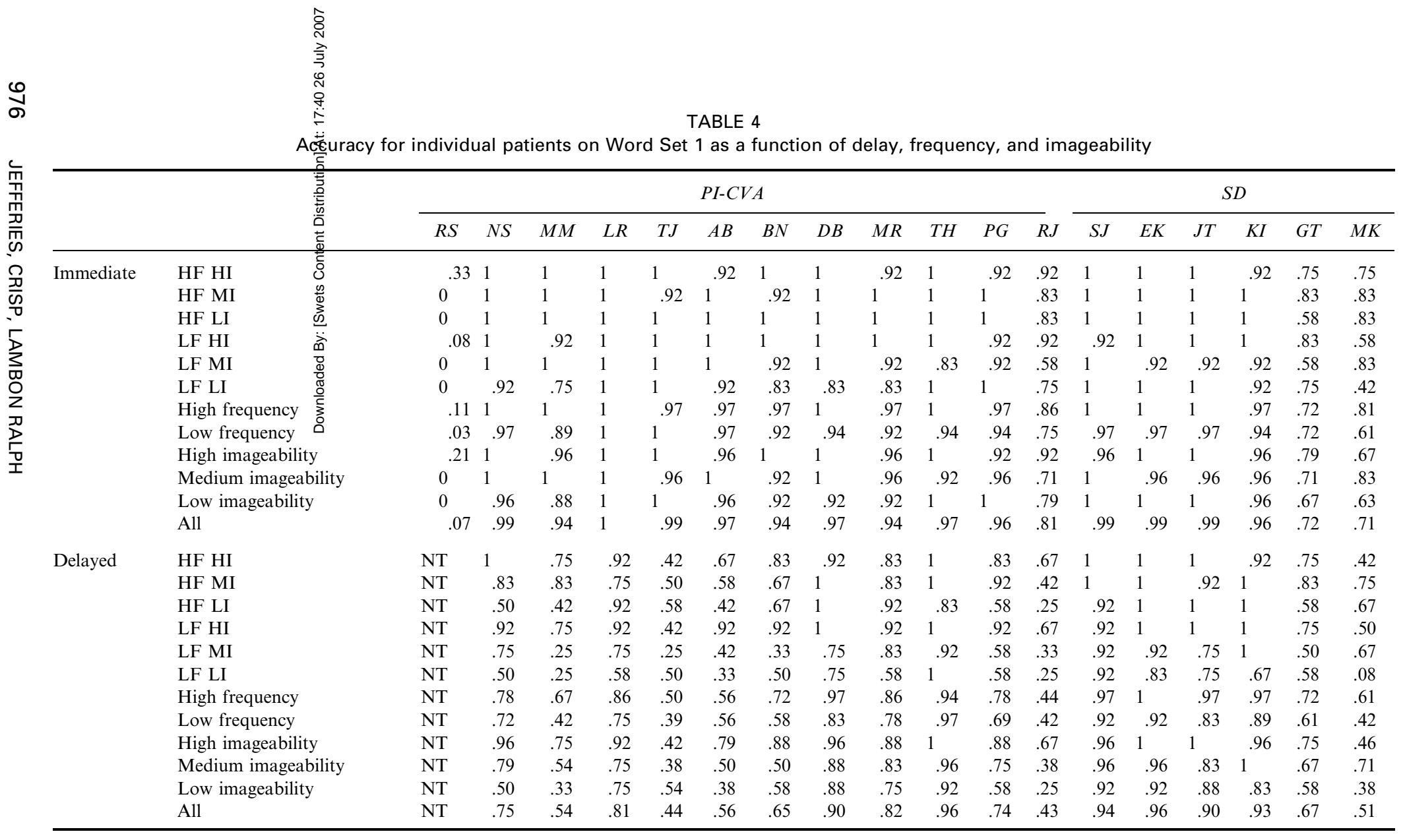

Figures show proportion of items correct. PI-CVA = phonologically impaired cerebrovascular accident patient. SD = semantic dementia. $\mathrm{HF}=$ high frequency, LF $=$ low frequency, $\mathrm{HI}=$ high imageability, $\mathrm{MI}=$ medium imageability, $\mathrm{LI}=$ low imageability. $\mathrm{NT}=$ not tested. 
affected by the delay, $F_{1}(2,30)=3.8, p=.03 ; F_{2}(2,66)=4.1, p=.02$. The frequency by imageability interaction reached significance for delayed repetition, $F_{l}(2$, $32)=8.7, p=.001 ; F_{2}(2,66)=5.8, p=.005$, but not immediate repetition, $F_{l}(2$, $32)=2.7, p=.09 ; F_{2}(2,66)=1.4, n s$.

Words: Set 2. The six SD patients and seven of the PI-CVA patients provided data on an additional set of words. A comparison of the two groups replicated many of the findings for Word Set 1 (see Table 5, which shows the mean performance for each group, and Table 6, which shows the outcome of analyses by subjects and items). Both groups showed poorer repetition after a delay; however, the delay had a greater

TABLE 5

Accuracy for each patient group on Word Set 2

\begin{tabular}{|c|c|c|c|c|}
\hline & \multicolumn{2}{|c|}{$S D(\mathrm{~N}=6)$} & \multicolumn{2}{|c|}{$\begin{array}{l}\text { Phonologically impaired } C V A \\
\qquad(\mathrm{~N}=5)\end{array}$} \\
\hline & Immediate & Delayed & Immediate & Delayed \\
\hline High frequency, high imageability & $.99(0.41)$ & $.99(0.41)$ & $.99(0.38)$ & $.94(1.07)$ \\
\hline High frequency, low imageability & $.89(1.76)$ & $.86(1.67)$ & $.94(1.07)$ & $.72(2.97)$ \\
\hline Low frequency, high imageability & $.92(2.04)$ & $.83(1.97)$ & $.99(0.38)$ & $.84(1.80)$ \\
\hline Low frequency, low imageability & $.80(3.31)$ & $.70(3.54)$ & $.91(1.50)$ & $.70(4.10)$ \\
\hline High frequency & .94 & .92 & .96 & .83 \\
\hline Low frequency & .86 & .77 & .95 & .77 \\
\hline High imageability & .95 & .91 & .99 & .89 \\
\hline Low imageability & .84 & .78 & .92 & .71 \\
\hline All & .90 & .85 & .96 & .80 \\
\hline
\end{tabular}

Figures show mean proportion of items correct for each group (standard deviation in parentheses).

TABLE 6

Analysis of differences in repetition accuracy between the two patient groups for Word Set 2

\begin{tabular}{llll}
\hline & $\begin{array}{c}\text { Semantic dementia } \\
(\mathrm{N}=6)\end{array}$ & $\begin{array}{c}\text { Phonologically impaired } \\
\text { CVA }(\mathrm{N}=7)\end{array}$ & $\begin{array}{c}\text { Cross-group comparison } \\
(\mathrm{N}=13)\end{array}$ \\
\hline Delay & $F_{1}=27.0, p=.01$ & $F_{1}=11.5, p=.01$ & $F_{1}=4.0, p=.07$ \\
& $F_{2}=7.4, p=.01$ & $F_{2}=52.6, p=.0001$ & $F_{2}=19.3, p=.0001$ \\
Frequency & $F_{1}=6.1, p=.06$ & $F_{1}=4.7, p=.07$ & $F_{1}=2.8, n s$ \\
& $F_{2}=13.6, p=.001$ & $F_{2}=2.4, n s$ & $F_{2}=4.7, p=.03$ \\
Frequency by delay & $F_{1}=30.0, p=.01$ & $F_{1}=1.1, n s$ & $F_{1}<1$ \\
& $F_{2}=2.3, n s$ & $F_{2}=1.1, n s$ & $F_{2}<1$ \\
Imageability & $F_{1}=6.3, p=.05$ & $F_{1}=7.6, p=.03$ & $F_{1}<1$ \\
& $F_{2}=12.2, p=.001$ & $F_{2}=21.2, p=.0001$ & $F_{2}<1$ \\
Imageability by delay & $F_{1}=1.0, n s$ & $F_{1}=5.7, p=.05$ & $F_{1}=2.4, p=.15$ \\
& $F_{2}<1$ & $F_{2}=5.1, p=.03$ & $F_{2}=4.4, p=.04$ \\
Frequency by imageability & $F_{1}<1$ & $F_{1}<1$ & $F_{1}<1$ \\
& $F_{2}<1$ & $F_{2}<1$ & $F_{2}<1$ \\
\hline
\end{tabular}

$F_{1}$ denotes analysis by subjects (degrees of freedom $=(1,5)$ for semantic dementia group; $(1,6)$ for phonologically impaired CVA group; $(1,11)$ for cross-group comparison). $F_{2}$ denotes analysis by items (degrees of freedom $=(1,52)$ for all comparisons). $n s=$ not significant $($ all $p>.1)$. 
impact on the PI-CVA patients. Both groups also showed effects of frequency and imageability (although the frequency effect was only marginally significant for the PI-CVA group). In the by-participants analysis, the SD group showed an interaction between frequency and delay. The delay impaired the SD patients' repetition of lowfrequency words that were presumably semantically degraded, Bonferroni $t_{1}(5)=5.8, p=.004$, but did not affect the repetition of high-frequency words, Bonferroni $t_{l}(5)=2.2, n s$. There was no interaction between frequency and delay for the PI-CVA patients. For the PI-CVA but not the SD patients, there was an interaction between imageability and delay. The delay had a larger effect on the PICVA patients' repetition of low-imageability words, Bonferroni $t_{l}(6)=3.2, p=.04$; $t_{2}(27)=6.0, p=.0001$, although there was also a significant effect of delay for highimageability words, Bonferroni $t_{1}(6)=3.1, p=.04 ; t_{2}(27)=4.0, p=.001$.

Words vs nonwords. A total of 11 PI-CVA patients and 6 SD patients performed immediate and delayed repetition of 48 nonwords matched to the low-frequency words from Set 1 . The group results are shown in Figure 2 and individual patient data are shown in Table 7 . In the complete set of items collapsing across syllable length, lexicality had a highly significant effect on the repetition of the PI-CVA group, $F_{1}(1,10)=136.2, p<.0001 ; F_{2}(1,94)=99.9, p<.0001$, and a somewhat smaller influence for the SD patients, $F_{l}(1,5)=2.6, n s ; F_{2}(1,94)=18.2, p<.0001$. The interaction between lexicality and patient group reached significance, $F_{l}(1$, $15)=3.5, p=.08 ; F_{2}(1,94)=16.2, p=.0001$. There was an interaction between lexicality and delay for the PI-CVA patients, $F_{l}(1,10)=16.9, p=.002 ; F_{2}(1$, $94)=30.9, p<.0001$, which reflected the fact that the delay impaired the repetition of nonwords, Bonferroni $t_{l}(10)=9.2, p<.0001 ; t_{2}(47)=19.5, p<.0001$, more substantially than the repetition of words, Bonferroni $t_{l}(10)=5.6, p=.0004$; $t_{2}(47)=9.9, p<.0001$. In contrast, the lexicality effect did not vary with the delay for the SD patients, $F_{l}(1,5)<1 ; F_{2}(1,94)<1$. The three-way interaction between

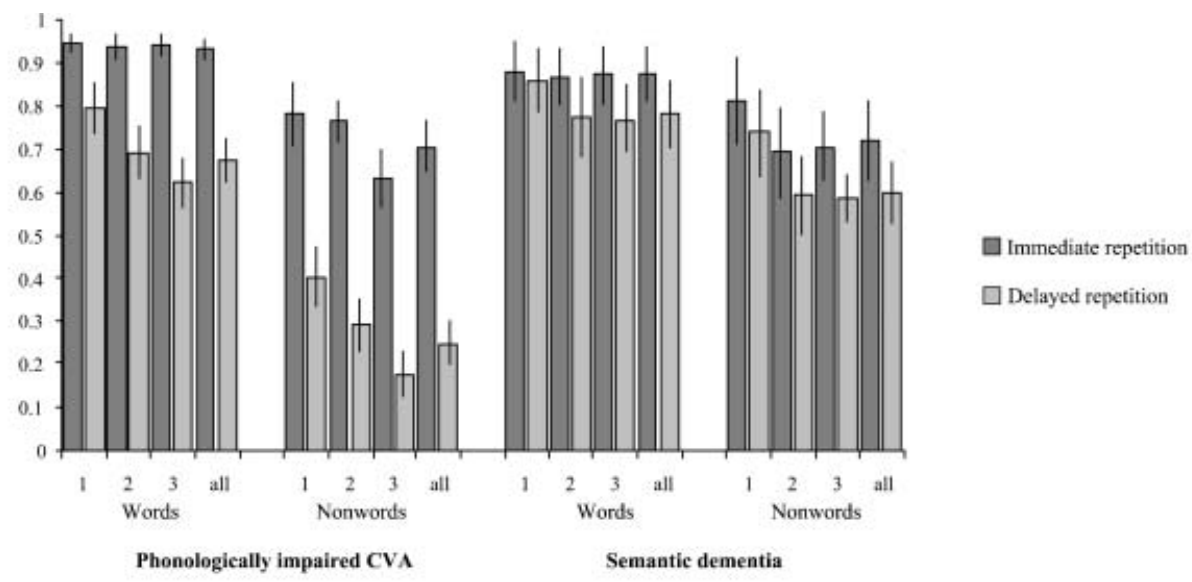

Note: Each set of bars shows immediate and delayed repetition for words/nonwords at three syllable lengths and combining across length. Error bars show standard error of mean.

Figure 2. Lexicality and item length effects for the two patient groups. 


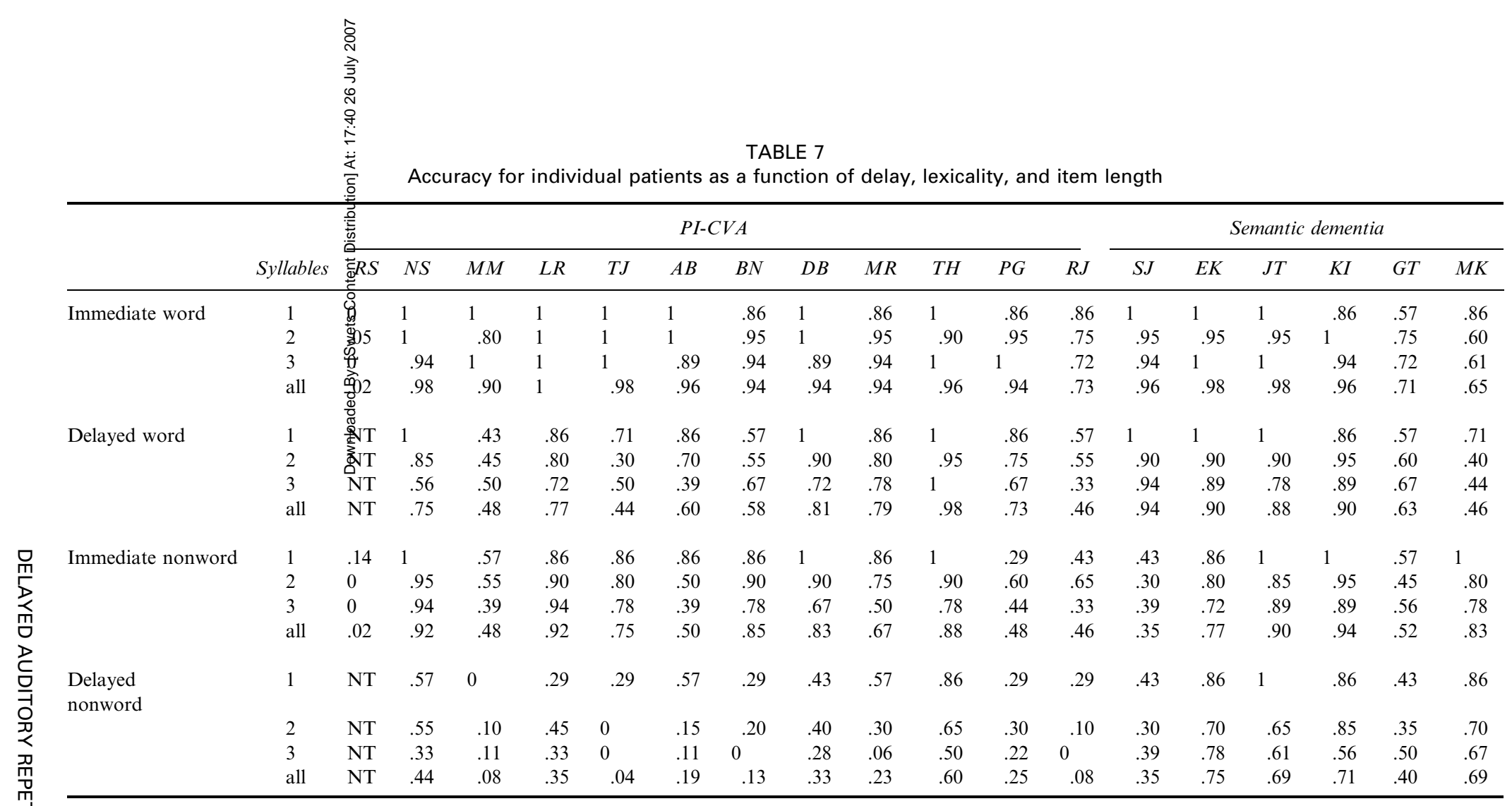

Figures show proportion of items correct. PI-CVA = phonologically impaired cerebrovascular accident patient. NT = not tested. 
patient group, lexicality, and delay reached significance, reflecting this difference between the two groups, $F_{1}(1,15)=5.4, p=.03 ; F_{2}(1,94)=13.5, p=.0004$.

Items contained one syllable $(N=7)$, two syllables $(N=20)$, three syllables $(N=18)$, or four syllables $(N=3)$. We considered the effect of syllable length on the repetition of nonwords, excluding the small number of items with four syllables from the analysis. The number of syllables had a significant effect on repetition accuracy for both the PI-CVA patients, $F_{l}(2,20)=10.1, p=.001 ; F_{2}(2,42)=3.9, p=.03$, and the SD patients, $F_{l}(2,10)=7.1, p=.01 ; F_{2}(2,42)<1$. There was no interaction between syllable length and patient group, $F_{l}(2,30)=1.9, n s ; F_{2}(2,42)=1.5$, $n s$.

\section{Accuracy: Correlations with composite semantic and phonological scores}

For each patient, the size of the lexicality, frequency, and imageability effects in delayed repetition were estimated by determining the difference between words/ nonwords, high/low-frequency words and high/low-imageability words (mediumimageability words were discarded). Similarly, we computed the difference between immediate/delayed repetition to examine the effect of the delay for words and nonwords. Correlations were calculated between the effect size for each patient and the composite measures of semantic and phonological impairment. This analysis included 11 PI-CVA patients and 6 SD patients who were tested on Word Set 1 (although we were unable to obtain a composite phonological score for one SD case, patient MK). The significance values reported below are one-tailed. Positive correlations indicate an association between good semantics/phonology and a large effect of the variable in question, whereas negative correlations indicate that semantic/phonological impairment was coupled with a large effect of the variable.

There was a significant negative correlation between the semantic and phonological composite measures, presumably indicating that the semantically impaired SD patients were generally good at the phonological tasks, whereas the phonologically impaired CVA patients had relatively good semantics $(r=-.43$, $p=.05)$. Good performance on semantic tasks was associated with a large influence of lexicality in delayed repetition $(r=.81, p<.0001)$. This correlation was significant for the SD group alone, despite the small sample size, indicating that the milder SD patients showed larger lexicality effects $(N=6, r=.92, p=.009)$. Semantic deficits were linked to large frequency effects both for all of the cases combined $(r=-.40$, $p=.05)$ and the SD group separately $(r=-.84, p=.03)$. Finally, combining the two groups, there was a correlation between semantic abilities and delay effects in nonword repetition $(r=.43, p=.04)$, reflecting the SD patients' insensitivity to delay.

Preserved phonological skills were linked to a reduced effect of delay on both word repetition $(r=-.50, p=.02)$ and nonword repetition $(r=-.36, p=.08)$. Patients with poor phonological skills also showed a more substantial effect of imageability $(r=-.63, p=.004)$.

\section{Errors}

Errors that were immediately and spontaneously self-corrected were excluded from this analysis. Incorrect responses were classified in the following way: Semantic errors were semantically or associatively related to the target word (e.g., KITTEN $\rightarrow$ 
"mouse"). Derivational errors included both inflectional errors (PRIVATE $\rightarrow$ "privately") and completions (WINDOW $\rightarrow$ "window cleaner"). There were two categories of phonological error. Close phonological errors shared at least $50 \%$ of the target phonemes (e.g., SUFFIX $\rightarrow$ "sussex"). Distant phonological errors shared at least one phoneme with the target, excluding the neutral schwa sound (ARBITOR $\rightarrow$ "abudy"). Formal (i.e., real word) errors were coded separately from nonword paraphasias, making it possible to consider influences on phonological errors (combining words and nonwords) and lexicality of responses (combining phonological and unrelated responses). A small number of errors were both phonologically and semantically related to the target words (WICKET $\rightarrow$ "cricket"). These are combined with the other semantic errors in the analysis below. Perseverations occurred when a previous response was repeated (e.g., Trial 1: QUAKE $\rightarrow$ "quake"; Trial 2: CRUSH $\rightarrow$ "quake"). A second category of incomplete or altered perseverations comprised responses that contained a significant degree of perseveration (e.g., Trial 1: STRENGTH $\rightarrow$ "strength"; Trial 2: CONSTANT $\rightarrow$ "struh"). Omissions were failures to respond, or responses such as "forgot it". Unrelated errors did not fall into any of these categories. The lexicality of close/ distant phonological errors and unrelated responses was recorded.

Word Set 1. This analysis is based on the matched subset of words $(N=72)$ discussed above. There were too few errors in immediate repetition to conduct a formal analysis. Only one PI-CVA patient (PS) made a substantial number of errors in this task - these were primarily omissions. Two SD patients (GT and MK) also had accuracy below $80 \%$ - most of their errors were close phonological approximations. The other patients in both groups made small numbers of close phonological errors.

Figure 3 shows average errors of each type as a proportion of total errors in delayed repetition for each patient group. Phonological errors (combining across the close and distant categories) were more numerous for the SD than the PI-CVA

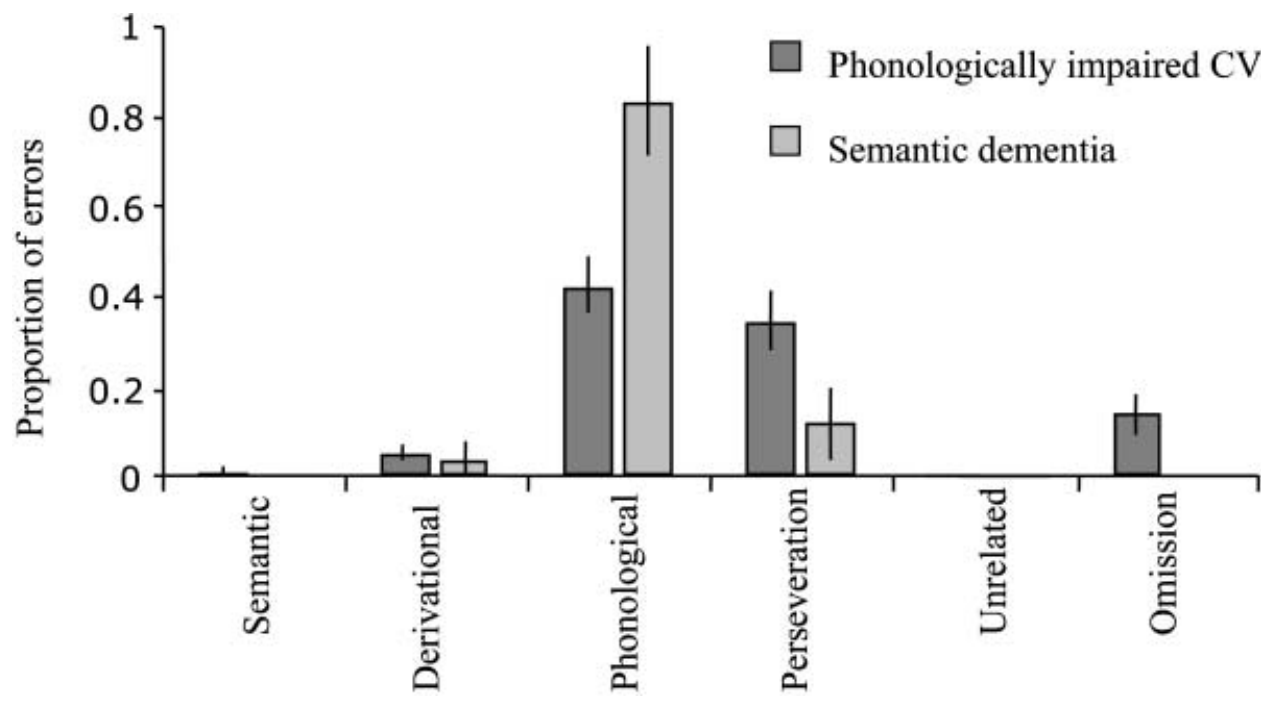

Figure 3. Errors on delayed repetition of Word Set 1. Errors are expressed as a proportion of total errors. Error bars show standard error of the mean. 
patients, $t(15)=3.33, p=.005$. The PI-CVA patients made more semantic/ derivational errors than the SD patients, $t(10.5)=2.82, p=.02$; collapsing these two categories. Thus semantic impairment was associated with relatively large numbers of phonological errors, whereas phonological impairment produced semantic/derivational errors (although it should be emphasised that semantic errors were a small proportion of the total number of errors and were only made by 3 out of 11 participants - in contrast, derivational errors were made by 8 of the CVA cases). Perseverations (combining the complete and partial perseveration categories) were also more frequent for the PI-CVA group, $t(15)=2.07, p=.06$, as were omissions, $t(15)=2.55, p=.02$. The majority of perseverations were complete repetitions of a previous response $(83 \%)$. Although the number of errors of some types was small, these effects were all significant in nonparametric tests (Mann Whitney $U<12.5, p<.04$ ). The pattern of results was also unchanged when errors were expressed as a proportion of items presented (as opposed to a proportion of total errors).

The PI-CVA patients made more real word responses in delayed word repetition than the SD patients: $40 \%$ vs $18 \%$ of phonological and unrelated errors; $\chi^{2}(1)=8.50$, $p=.004$. This difference may have reflected the PI-CVA patients' increased reliance on semantics in the repetition task.

Correlations between the number of errors in each category (expressed as a proportion of total errors) and the composite measures of semantic and phonological impairment were calculated, combining the two patient groups. There was a negative correlation between the phonological factor and semantic/derivational errors $(r=-.49$, two-tailed $p=.06)$, indicating that these errors were associated with phonological impairment. These errors were also associated with poor nonword repetition, both immediately $(r=-.61$, two-tailed $p=.01)$ and after a delay $(r=-.67$, two-tailed $p=.004)$. There was no association between semantic/derivational errors and the semantic factor $(r=.32$, two-tailed $p=.21)$. There was a strong negative correlation between the semantic factor and phonological errors $(r=-.76$, two-tailed $p=.0004)$, indicating that phonological errors were associated with semantic impairment. Conversely, there was a positive correlation between the phonological factor and phonological errors $(r=.51$, two-tailed $p=.04)$, indicating that the patients with good phonology (i.e., the SD patients) typically made more phonological errors. Phonological impairment was associated with a higher incidence of omission errors $(r=-.85$, two-tailed $p<.0001)$. In addition, semantically impaired patients made fewer perseverative errors $(r=.61$, two-tailed $p=.009)$, presumably reflecting the fact that these errors were uncommon amongst the SD patients.

Word Set 2. An analysis of delayed repetition errors for Word Set 2 replicated many of these findings (see Table 8). The SD group made more phonological errors than the PI-CVA patients, $t(11)=4.90, p=.0005$. The PI-CVA patients made more perseverations, $t(11)=2.48, p=.03$, and showed a trend towards making more omissions, $t(6)=2.10, p=.08$. The difference in phonological errors reached significance in a nonparametric test (Mann-Whitney $U=1.0 ; p=.004$ ). The difference in perseverations approached significance (Mann-Whitney $U=8.0$; $p=.06$ ). However, there was no significant difference between the groups in the number of semantic/derivational errors due to the small number of errors of this type, $t(11)<1$; Mann-Whitney $U=16.0, p=.4$. 
TABLE 8

Errors on delayed repetition of Word Set 2

\begin{tabular}{lccccc}
\hline & \multicolumn{2}{c}{$P I-C V A(\mathrm{~N}=7)$} & & \multicolumn{2}{c}{ Semantic dementia $(\mathrm{N}=6)$} \\
\cline { 2 - 3 } \cline { 5 - 6 } & $M$ & $S D$ & & $M$ & $S D$ \\
\hline Semantic \& derivational & .16 & .37 & & .04 & .10 \\
Phonological & .31 & .18 & & .86 & .22 \\
Perseverations & .41 & .28 & & .10 & .15 \\
Unrelated & .02 & .05 & & 0 & 0 \\
Omission & .10 & .12 & & 0 & 0 \\
\hline
\end{tabular}

Errors are expressed as a proportion of total errors. Figures show mean and standard deviation for each group. PI-CVA = phonologically impaired cerebrovascular accident patient.

Nonwords. Table 9 shows the errors made by the two patient groups in immediate and delayed nonword repetition. One PI-CVA patient, RS, was not tested on the delayed repetition task and was excluded from this analysis. In immediate repetition, the errors made by both groups were almost exclusively phonological in nature (although patient RS made a larger number of errors in immediate repetition, $60 \%$ of which were omissions). In delayed repetition, the majority of errors were again phonological. This category represented a larger proportion of the total number of errors for SD patients compared with PI-CVA patients, $t(15)=2.74, p=.02$; MannWhitney $U=3.0, p=.002$. The PI-CVA group made more omissions, $t(15)=2.95$, $p=.01$; Mann-Whitney $U=0.0, p=.001$, and perseverations, $t(15)=1.96, p=.07$;

TABLE 9

Errors on immediate and delayed repetition of nonwords

\begin{tabular}{|c|c|c|c|c|c|c|}
\hline & & & \multicolumn{2}{|c|}{$\begin{array}{l}P I-C V A \\
(\mathrm{~N}=11)\end{array}$} & \multicolumn{2}{|c|}{$\begin{array}{l}\text { Semantic dementia } \\
\qquad(\mathrm{N}=6)\end{array}$} \\
\hline & & & $M$ & $S D$ & $M$ & $S D$ \\
\hline \multirow[t]{10}{*}{ Nonwords } & \multirow[t]{5}{*}{ Immediate } & Semantic \& derivational & 0 & 0 & 0 & 0 \\
\hline & & Phonological & .97 & .08 & 1 & 0 \\
\hline & & Perseveration & 0 & 0 & 0 & 0 \\
\hline & & Unrelated & 0 & 0 & 0 & 0 \\
\hline & & Omission & .03 & .08 & 0 & 0 \\
\hline & \multirow[t]{5}{*}{ Delayed } & Semantic \& derivational & 0 & 0 & 0 & 0 \\
\hline & & Phonological & .72 & .23 & .99 & .03 \\
\hline & & Perseveration & .19 & .21 & .01 & .03 \\
\hline & & Unrelated & .01 & .03 & 0 & 0 \\
\hline & & Omission & .08 & .06 & 0 & 0 \\
\hline \multirow{5}{*}{$\begin{array}{l}\text { Words } \\
\text { (matched } \\
\text { subset) }\end{array}$} & \multirow[t]{5}{*}{ Delayed } & Semantic \& derivational & .10 & .11 & .06 & .14 \\
\hline & & Phonological & .52 & .25 & .89 & .27 \\
\hline & & Perseveration & .22 & .21 & .06 & .14 \\
\hline & & Unrelated & .01 & .03 & 0 & 0 \\
\hline & & Omission & .15 & .14 & 0 & 0 \\
\hline
\end{tabular}

Errors are expressed as a proportion of total errors. Figures show mean and standard deviation for each group. PI-CVA = phonologically impaired cerebrovascular accident patient. 
Mann-Whitney $U=12.5, p=.04$. The majority of perseverations were partial ( $84 \%$; e.g., pomor $\rightarrow$ "porg"; gintry $\rightarrow$ "forg").

Effect of lexicality. Patients' errors on nonwords and the matched subset of words were compared (see Table 9). The SD patients' errors on both delayed word and nonword repetition were almost exclusively phonologically related to the target $(97 \%$ and $99 \%$ of errors respectively, combining across the patients). The fact that this group did not show any differences in the errors they made to words and nonwords might have reflected their severe semantic impairments, with the repetition of unknown words resembling that of nonwords. In contrast, the PI-CVA group made more semantic errors in word than nonword repetition, $t(10)=3.00, p=.01$; Wilcoxon Signed Ranks Test $Z=2.52, p=.01$, and more phonological errors in nonword than word repetition, $t(10)=2.58, p=.03$; Wilcoxon Signed Ranks Test $\mathrm{Z}=2.05, p=.04$. They also showed a trend towards making more omission errors in nonword repetition, $t(10)=1.98, p=.08$; Wilcoxon Signed Ranks Test $Z=1.87$, $p=.06$.

Perseverative errors. It is somewhat surprising that the more phonologically impaired group, who showed poorer repetition following a delay, also made more perseverative responses in delayed repetition. We examined the perseverations of the PI-CVA patients during delayed repetition of the complete group of 96 items included in Word Set 1 in more detail. This analysis included perseverations that were spontaneously self-corrected. The number of perseverations per patient ranged from $2.1 \%$ to $44.8 \%$ of trials $(M=11.8 \% ; S D=13.0)$. On most of these trials, the perseveration occurred despite accurate immediate repetition (e.g., Trial 1: RIVER $\rightarrow$ "river"... "river"; Trial 2: HUMOUR $\rightarrow$ "humour"... "river"). A preceding target word was produced in its entirety in $70 \%$ of these perseverations; the remaining trials were either complete perseverations of an error (e.g., rogue $\rightarrow$ "curtains"; winter $\rightarrow$ "curtains") or were partial perseverations (e.g., window $\rightarrow$ window; helmet $\rightarrow$ "win"). Considering only complete perseverations of targets, $71 \%$ involved the repetition of high-frequency words, as opposed to $29 \%$ on which lowfrequency words were reproduced. These values significantly differed from the expected ratio of 1:1 (Binomial $p=.001$ ), suggesting that high-frequency words were more likely to be perseverated. However, highly imageable words were not more likely to be reproduced on subsequent trials (34\% high imageability; $18 \%$ medium imageability; 32\% low imageability). As well as considering the frequency/ imageability of perseverated items, we can also consider whether perseverations were more likely to occur for low-frequency or less imageable words. Perseverations, expressed as a percentage of incorrect trials, occurred at a rate of $47 \%$ for highfrequency words and 33\% for low-frequency words. The values for high-, medium-, and low-imageability words were $53 \%, 41 \%$, and $33 \%$ respectively. Therefore, perseverations did not occur at an elevated rate relative to overall accuracy for either low-frequency or low-imageability trials.

The majority of perseverations reflected the repetition of the immediately preceding response (74\%; e.g., river $\rightarrow$ river; humour $\rightarrow$ river). On $11 \%$ of trials, the penultimate response was repeated (e.g., river $\rightarrow$ river; humour $\rightarrow$ humour; master $\rightarrow$ river) and a further $6 \%$ of perseverations reproduced the response three trials back; $4 \%$ of perseverations were repetitions of items between four and ten trials back; and $5 \%$ of perseverations were over a lag of more than 10 trials. The majority 
of items were only produced in a single perseverative error (78\%). However, there were instances in which the same word was produced repeatedly: $19 \%$ of words were perseverated from two to four times and $4 \%$ of words were involved in more than four perseverative errors. The performance of one patient in particular (TJ) was characterised by strings of continuous perseveration.

The proportion of each PI-CVA patient's errors that were perseverative did not correlate with the composite measures of semantics $(r=.15, N=11, p=.6)$ or phonology $(r=.01, N=11, p=.99)$. Therefore, although perseverations characterised the performance of the PI-CVA group who were relatively phonologically impaired/semantically intact, the frequency of these errors did not appear to relate to these capacities. We will consider why the PI-CVA patients made more numerous perseverations in the General Discussion.

\section{GENERAL DISCUSSION}

This work investigated the impact of phonological and semantic impairment on immediate and delayed repetition in CVA and semantic dementia (SD). Lexicality, frequency, and imageability were varied in order to manipulate the extent to which lexical-semantic knowledge could act to constrain and maintain phonological activation. If semantic memory and phonology underpin repetition in an interactive way as envisaged by single route theories (Dell et al., 1997; Foygel \& Dell, 2000; Patterson et al., 1994; Plaut \& Kello, 1999), the degree of semantic and phonological impairment should interact with experimental factors purported to tap semantic and phonological processing. Our findings supported this hypothesis.

The phonologically impaired CVA patients showed relatively good immediate repetition but much poorer performance after a delay. This finding is consistent with the view that delay-dependent phonological decay or interference exacerbates the effects of phonological impairment. The PI-CVA patients also showed substantial effects of lexicality and imageability that were larger in delayed than immediate repetition; this result suggests that lexical-semantic knowledge plays a greater role in repetition when the contribution of phonology is minimised due to brain damage or the nature of the task. The semantically impaired SD patients, on the other hand, showed smaller effects of lexicality and imageability in repetition, reflecting the reduced impact of lexical-semantic processing in this condition. For these patients, the delay had a more substantial effect on the repetition of poorly understood, lowfrequency words relative to high-frequency words that were better understood, suggesting that phonology plays a critical role in the repetition of items not adequately supported by lexical-semantic representations. A complementary finding was that phonological and not semantic impairment led to an increase in the number of semantic/derivational errors in repetition. It seems likely that these errors reflected the predominance of semantic processing in the PI-CVA group. Conversely, semantic impairment increased the incidence of phonological errors, in line with the hypothesis that semantic representations help to constrain phonological activation (Patterson et al., 1994).

The "semantic-phonological" computational model of Foygel and Dell (2000) is able to capture many of these findings (see http://langprod.cogsci.uiuc.edu/cgi-bin/ webfit.cgi). This model incorporates three layers of phonological, lexical, and semantic nodes linked by bidirectional connections. Activation across all of these nodes decays at a predetermined rate. Variation between aphasic patients is 
accommodated by altering two parameters, the strength of semantic and phonological connections. Specific damage to the phonological-lexical weights produces rapidly decreasing repetition performance. As the level of phonological impairment is increased, repetition shows an increasing influence of semantic memory: the errors change from phonologically related nonwords to include a larger number of real words that are sometimes semantically related to the target. Therefore, the model successfully accommodates the correlation that we observed between phonological impairment and semantic errors in repetition. If the number of steps between the initial activation and the output is increased to simulate delayed repetition (as in Martin et al., 1996), semantic errors emerge with milder phonological problems: this is also consistent with our data. When the semanticlexical weights are specifically impaired in a model with intact phonology (perhaps reflecting the pattern in semantic dementia ${ }^{6}$ ), there is very little impact on immediate repetition (accuracy remains at $96 \%$ even when the semantic weights are set to zero). However, delayed repetition is more substantially impaired by this selective impairment of semantic memory, in line with our findings. A combination of semantic and phonological damage in this model produces a more striking impairment of repetition, demonstrating the interactive nature of semantic and phonological processing. An extension of the model might also be able to account for the interactions that we observed between task variables (e.g., lexicality, frequency, imageability, and delay) and the degree of semantic and phonological damage. Nevertheless, some of our findings do not fit comfortably within this framework: the model is only concerned with single trials and therefore cannot explain perseverations. It also appears that the model cannot fit the pattern of errors shown by SD patients in naming and delayed repetition. In picture naming, as acknowledged by Foygel and Dell, the model greatly underestimates the rate of semantic errors and predicts the presence of phonologically related words and unrelated responses, which rarely occur for SD patients. Additionally, in delayed repetition, the model overestimates the proportion of errors that are real words for SD patients.

The pattern of performance displayed by the PI-CVA group, i.e., large effects of lexicality and imageability in repetition, together with a predominance of phonological errors, has been termed "phonological dysphasia" (Hanley \& Kay, 1997; Hanley et al., 2002), in order to distinguish it from the "deep dysphasic" syndrome characterised by semantic errors in repetition. It is interesting to note that in this study the PI-CVA patients began to make semantic as well as phonological errors when repetition was delayed, suggesting that these two disorders may lie on a continuum of impairment (as has been suggested in the reading domain for phonological and deep dyslexia: Crisp \& Lambon Ralph, 2006; Friedman, 1996). Cases with more severe phonological problems might generate semantic errors in repetition, whereas more mildly impaired patients might only produce phonological errors. The deep dysphasic patient studied by N. Martin and colleagues (1996) resolved into a phonological dysphasia during recovery, in line with this view. The presence of a delay in the repetition task apparently aggravated the patients'

\footnotetext{
${ }^{6}$ The semantic impairment in SD is multimodal: the meanings of words are degraded (corresponding to damage of lexical-semantic weights) but other modalities of input are equally affected suggesting damage to the semantic representations themselves. This type of damage is not incorporated in these computational implementations of the interactive activation model.
} 
phonological problems, increasing their reliance on lexical-semantic activation. The emergence of semantic errors, therefore, might have reflected the normal operation of the lexical-semantic system unconstrained by phonological activation that would otherwise have inhibited semantic neighbours of the target. In our study, semantic/ derivational errors correlated with phonological and not semantic abilities, supporting this viewpoint. Hanley and Kay (1997) have also suggested that phonological as opposed to semantic errors occur in repetition when there is partial preservation of the non-lexical (phonological) route.

The source of the imageability effect in deep/phonological dysphasia remains a point of contention. Hanley and Kay (1997) proposed that two separate impairments to both the non-lexical and lexical-semantic routes underlie this disorder. By this view, substantial imageability effects reflect the impairment of the lexical-semantic route. In the current study, however, the size of the imageability effect was correlated with phonological and not semantic impairment. Martin and Saffran (1997) obtained a similar finding and showed how it could be elegantly accommodated by an interactive, single-route model in which lexical-semantic processing plays a greater role in repetition in the face of phonological impairment. Our findings build on this study by demonstrating that lexical-semantic factors such as lexicality, frequency, and imageability not only interact with the degree of semantic and phonological impairment in a neuropsychological population, but also interact with the presence of a delay in the repetition task and influence the types of errors that are made.

One issue that warrants further discussion is the circumstances in which semantic/ phonological impairment increases or decreases the effect of semantic/phonological variables in repetition. In the Introduction we noted that, in SD, exaggerated imageability effects might occur if highly imageable words are more resistant to semantic degradation by virtue of their richer representations; however, imageability effects might be reduced if the normal advantage for processing highly imageable words is eroded by the semantic impairment. According to the interactive framework, there should be reduced effects of semantic variables (and enhanced effects of phonological variables) in the face of semantic impairment, as processing shifts away from the semantic units, which are sensitive to these variables, and draws more heavily on phonology. For phonological impairment, the reverse should be true. In the present study, there are several examples of this type of interactivity. The SD patients showed a smaller imageability effect than the PI-CVA group overall, and the impact of the delay was larger for low-frequency and low-imageability items. However, the SD patients also showed a substantial frequency effect and the PICVA patients were more severely affected by the delay than the SD group. In some situations, therefore, semantic/phonological impairment can result in exaggerated effects of variables that purportedly tap these processes. We have argued that the increased delay effect for the PI-CVA group occurred because immediate repetition is not a highly phonologically demanding task, whereas delayed repetition exacerbates the effects of phonological impairment. Similarly, SD patients almost universally show large effects of frequency and it is thought that this is because lowfrequency words and concepts degrade at an earlier stage of the condition (Funnell, 1995). It is interesting to note that Martin and Saffran (1997) found a positive and often non-significant relationship between semantic ability and frequency in a group of CVA patients, whereas we observed a significant negative correlation: the semantically impaired SD patients showed larger not smaller effects of frequency. This difference might have reflected the nature of the comprehension impairment in 
the two groups: although high-frequency items might be more robust in the face of semantic degradation in SD, frequency effects may be weakened in comprehensionimpaired CVA patients who do not have degradation of the semantic representations themselves, but instead show difficulty accessing semantics effectively (Warrington \& Cipolotti, 1996).

Other differences in the repetition performance of the SD and PI-CVA patients might have stemmed from the distinct aetiologies involved. The PI-CVA patients made significantly more perseverative errors than the SD patients, despite their difficulties in delayed repetition. Indeed, all of the PI-CVA cases made at least some perseverative errors, although the rates of perseveration were highly variable. In contrast, this error type almost never occurred in the SD group. One possible explanation of this group difference is that perseverations arose from the PI-CVA patients' relatively intact and durable semantic memory combined with poor processing of phonological input, which prevented new items from overriding the lingering activation of older items. Two findings speak against this view, however: (1) the proportion of errors that were perseverative did not correlate with either semantic or phonological abilities; (2) substantial perseveration was observed in both word and nonword repetition. An alternative possibility is that perseverations arise in CVA but not SD because of differences in the nature of the underlying brain damage. Several researchers have argued that perseverations are the product of a neuromodulatory failure resulting from low levels of acetylcholine, which purportedly makes cells more sensitive to feed-forward input (Gotts, della Rocchetta, \& Cipolotti, 2002; Sandson \& Albert, 1987). In perseverative CVA patients, there may be a cholinergic deficit that prevents new inputs from overriding current processing. Subcortical involvement is comparatively common in stroke, potentially producing deficits of this nature: in contrast, subcortical regions are relatively well preserved in SD (Galton et al., 2001; Mummery, Patterson, Price, Ashburner, Frackowiak, \& Hodges, 2000).

Although we acknowledge that the nature of the underlying brain damage is likely to have important behavioural consequences, we observed evidence of substantial interactivity between semantics and phonology in the repetition performance of both stroke and semantic dementia patients. The PI-CVA patients showed sizeable effects of lexicality and imageability that were larger in delayed than immediate repetition, whereas the semantically impaired SD patients showed a reduced influence of these lexical-semantic variables and a delay effect that was larger for more poorly comprehended low-frequency items. In addition, patients with severe phonological deficits in the context of nonfluent progressive aphasia can resemble the phonologically impaired CVA cases reported here. Tree, Perfect, Hirsh, and Copstick (2001) described one such progressive aphasic patient who showed strong influences of lexicality/imageability and made semantic errors in repetition. Therefore, converging evidence for the interactive account of repetition is provided by patients with both fluent (SD) and non-fluent progressive aphasia, as well as CVA cases.

\section{REFERENCES}

Baayen, R. H., Piepenbrock, R., \& van Rijn, H. (1993). The CELEX Lexical Database. [CD-ROM]. Philadelphia, PA: Linguistic Data Consortium, University of Pennsylvania. 
Bozeat, S., Lambon Ralph, M. A., Patterson, K., Garrard, P., \& Hodges, J. R. (2000). Non-verbal semantic impairment in semantic dementia. Neuropsychologia, 38, 1207-1215.

Caza, N., Belleville, S., \& Gilbert, B. (2002). How loss of meaning with preservation of phonological word form affects immediate serial recall performance: A linguistic account. Neurocase, 8, 255-273.

Crisp, J., \& Lambon Ralph, M. A. (2006). Unlocking the nature of the phonological-deep dyslexia continuum: The keys to reading aloud are in phonology and semantics. Journal of Cognitive Neuroscience, 18, 348-362.

Croot, K., Patterson, K., \& Hodges, J. R. (1998). Single word production in non-fluent progressive aphasia. Brain and Language, 61, 226-273.

Dell, G. S., \& O’Seaghda, P. G. (1992). Stages of lexical access in language production. Cognition, 42, 287-314.

Dell, G. S., Schwartz, M. F., Martin, N., Saffran, E. M., \& Gagnon, D. A. (1997). Lexical access in aphasic and nonaphasic speakers. Psychological Review, 104, 801-838.

Forde, E. M. E., \& Humphreys, G. W. (2002). The role of semantic knowledge in short-term memory. Neurocase, 8, 13-27.

Foygel, D., \& Dell, G. S. (2000). Models of impaired lexical access in speech production. Journal of Memory and Language, 43, 182-216.

Franklin, S., Turner, J., \& Ellis, A. W. (1992). The ADA comprehension battery: Action for dysphasic adults. York, UK: York Human Neuropsychology Laboratory.

Friedman, R. B. (1996). Recovery from deep alexia to phonological alexia: Points on a continuum. Brain and Language, 52, 114-128.

Funnell, E. (1995). Objects and properties: A study of the breakdown of semantic memory. Memory, 3, 497-518.

Galton, C. J., Patterson, K., Graham, K., Lambon Ralph M., A., Williams, G., \& Antoun, N. et al. (2001). Differing patterns of temporal atrophy in Alzheimer's disease and semantic dementia. Neurology, 57, 216-225.

Gotts, S. J., della Rocchetta, A. I., \& Cipolotti, L. (2002). Mechanisms underlying perseveration in aphasia: Evidence from a single case study. Neuropsychologia, 40, 1930-1947.

Hanley, J. R., Dell, G. S., Kay, J., \& Baron, R. (2004). Evidence for the involvement of a nonlexical route in the repetition of familiar words: A comparison of single and dual route models of auditory repetition. Cognitive Neuropsychology, 21, 147-158.

Hanley, J. R., \& Kay, J. (1997). An effect of imageability on the production of phonological errors in auditory repetition. Cognitive Neuropsychology, 14, 1065-1084.

Hanley, J. R., Kay, J., \& Edwards, M. (2002). Imageability effects, phonological errors, and the relationship between auditory repetition and picture naming: Implications for models of auditory repetition. Cognitive Neuropsychology, 19, 193-206.

Hillis, A., \& Caramazza, A. (1991). Mechanisms for accessing lexical representations for output: Evidence from a category specific semantic deficit. Brain and Language, 40, 106-144.

Hodges, J. R., Patterson, K., Oxbury, S., \& Funnell, E. (1992). Semantic dementia: Progressive fluent aphasia with temporal lobe atrophy. Brain, 115, 1783-1806.

Howard, D., \& Franklin, S. (1988). Missing the meaning. Cambridge, MA: MIT Press.

Howard, D., \& Patterson, K. (1992). Pyramids and Palm Trees: A test of semantic access from pictures and words. Bury St. Edmunds, UK: Thames Valley Test Company.

Jefferies, E., Frankish, C., \& Lambon Ralph, M. A. (2006a). Lexical and semantic binding in verbal shortterm memory. Journal of Memory and Language, 54, 81-96.

Jefferies, E., Jones, R., Bateman, D., \& Lambon Ralph, M. A. (2004a). When does word meaning affect immediate serial recall in semantic dementia? Cognitive, Affective and Behavioral Neuroscience, 4, $20-42$.

Jefferies, E., Jones, R. W., Bateman, D., \& Lambon Ralph, M. A. (2005). A semantic contribution to nonword recall? Evidence for intact phonological processes in semantic dementia. Cognitive Neuropsychology, 22, 183-212.

Jefferies, E., Patterson, K., Bateman, D., Jones, R., \& Lambon Ralph, M. A. (2006b). The natural history of "pure" late-stage semantic dementia. Neurocase, 12, 1-14.

Jefferies, E., Patterson, K., Jones, R. W., Bateman, D., \& Lambon Ralph, M. A. (2004). A categoryspecific advantage for numbers in verbal short-term memory: Evidence from semantic dementia. Neuropsychologia, 42, 639-660.

Jones, G. V. (1985). Deep dyslexia, imageability and ease of prediction. Brain and Language, 24, 1-19. 
Katz, R. B., \& Goodglass, H. (1990). Deep dysphasia: Analysis of a rare form of repetition disorder. Brain and Language, 39, 153-185.

Kay, J., Lesser, R., \& Coltheart, M. (1992). Psycholinguistic Assessments of Language Processing in Aphasia. Hove, UK: Lawrence Erlbaum Associates Ltd.

Knott, R., Patterson, K., \& Hodges, J. R. (1997). Lexical and semantic binding effects in short-term memory: Evidence from semantic dementia. Cognitive Neuropsychology, 14, 1165-1216.

Knott, R., Patterson, K., \& Hodges, J. R. (2000). The role of speech production in auditory-verbal shortterm memory: Evidence from progressive fluent aphasia. Neuropsychologia, 38, 125-142.

Kucera, H., \& Francis, W. N. (1967). Computational analysis of present-day American English. Providence, RI: Brown University Press.

Lambon Ralph, M. A., Moriarty, L., \& Sage, K. (2002). Anomia is simply a reflection of semantic and phonological impairments: Evidence from a case-series study. Aphasiology, 16, 56-82.

Majerus, S., Lekeu, F., Van der Linden, M., \& Salmon, E. (2001). Deep dysphasia: Further evidence on the relationship between phonological short-term memory and language processing impairments. Cognitive Neuropsychology, 18, 385-410.

Martin, N., Dell, G. S., Saffran, E. M., \& Schwartz, M. F. (1994). Origins of paraphasias in deep dysphasia: Testing the consequences of a decay impairment to an interactive spreading activation model of lexical retrieval. Brain and Language, 47, 609-660.

Martin, N., \& Saffran, E. M. (1992). A computational account of deep dysphasia: Evidence from a single case study. Brain and Language, 43, 240-274.

Martin, N., \& Saffran, E. M. (1997). Language and auditory-verbal short-term memory impairments: Evidence for common underlying processes. Cognitive Neuropsychology, 14, 641-682.

Martin, N., Saffran, E., \& Dell, G. (1996). Recovery in deep dysphasia: Evidence for a relation between auditory-verbal STM capacity and lexical errors in repetition. Brain and Language, 52, 83-113.

Martin, R. C., \& Lesch, M. (1996). Associations and dissociations between language impairment and list recall: Implications for models of STM. In S. E. Gathercole (Ed.), Models of short-term memory (pp. 149-178). Hove, UK: Psychology Press.

Martin, R. C., Lesch, M. F., \& Bartha, M. C. (1999). Independence of input and output phonology in word processing and short-term memory. Journal of Memory and Language, 41, 3-29.

Martin, R. C., Shelton, J., \& Yaffee, L. S. (1994). Language processing and working memory: Neuropsychological evidence for separate phonological and semantic capacities. Journal of Memory and Language, 33, 83-111.

McCarthy, R., \& Warrington, E. K. (1984). A two-route model of speech production: Evidence from aphasia. Brain, 107, 463-485.

McCarthy, R. A., \& Warrington, E. K. (1987). The double dissociation of short-term memory for lists and sentences: Evidence from aphasia. Brain, 110, 1545-1563.

Michel, F., \& Andreewsky, A. (1983). Deep dysphasia: An analogue of deep dyslexia in the auditory modality. Brain and Language, 18, 212-223.

Mummery, C. J., Patterson, K., Price, C. J., Ashburner, J., Frackowiak, R. S. J., \& Hodges, J. R. (2000). A voxel-based morphometry study of semantic dementia: Relationship between temporal lobe atrophy and semantic memory. Annals of Neurology, 47, 36-45.

Patterson, K., Graham, N., \& Hodges, J. R. (1994). The impact of semantic memory loss on phonological representations. Journal of Cognitive Neuroscience, 6, 57-69.

Patterson, K., \& Marcel, A. J. (1992). Phonological ALEXIA or PHONOLOGICAL alexia? In J. Alegria, D. Holender, J. Junca de Morais, \& M. Radeau (Eds.), Analytic approaches to human cognition (pp. 259-274). Amsterdam: Elsevier.

Plaut, D. C., \& Kello, C. T. (1999). The emergence of phonology from the interplay of speech comprehension and production: A distributed connectionist approach. In B. MacWhinney (Ed.), The emergence of language. Mahwah, NJ: Lawrence Erlbaum Associates Inc.

Plaut, D. C., \& Shallice, T. (1991). Deep dyslexia: A case study in connectionist neuropsychology. Cognitive Neuropsychology, 10, 377-500.

Roodenrys, S., \& Hinton, M. (2002). Sublexical or lexical effects on serial recall of nonwords? Journal of Experimental Psychology: Learning, Memory and Cognition, 28, 29-33.

Sandson, J., \& Albert, M. L. (1987). Perseveration in behavioral neurology. Neurology, 37, 1736-1741.

Snowden, J. S., Goulding, P. J., \& Neary, D. (1989). Semantic dementia: A form of circumscribed cerebral atrophy. Behavioural Neurology, 2, 167-182. 
Snowden, J. S., Neary, D., \& Mann, D. M. A. (Eds.). (1996). Frontotemporal lobar degeneration: Frontotemporal dementia, progressive aphasia, semantic dementia. London: Churchill Livingstone.

Tree, J. J., Perfect, T. J., Hirsh, K. W., \& Copstick, S. (2001). Deep dysphasic performance in non-fluent progressive aphasia: A case study. Neurocase, 7, 473-487.

Treiman, R., \& Danis, C. (1988). Short-term memory errors for spoken syllables are affected by the linguistic structure of the syllables. Journal of Experimental Psychology: Learning, Memory and Cognition, 14, 145-152.

Valdois, S., Carbonnel, S., David, D., Rousset, S., \& Pellat, J. (1995). Confrontation of PDP models and dual-route models through the analysis of a case of deep dysphasia. Cognitive Neuropsychology, 12, 681-724.

Warrington, E. K., \& Cipolotti, L. (1996). Word comprehension: The distinction between refractory and storage impairments. Brain, 119, 611-625.

Warrington, E. K., McKenna, P., \& Orpwood, L. (1998). Single word comprehension: A concrete and abstract word synonym test. Neuropsychological Rehabilitation, 8, 143-154.

Wilshire, C. E., \& Fisher, C. A. (2004). "Phonological" dysphasia: A cross-modal phonological impairment affecting repetition, comprehension, and production. Cognitive Neuropsychology, 21, $187-210$.

Wilshire, C. E., \& McCarthy, R. A. (1996). Experimental investigations of an impairment in phonological encoding. Cognitive Neuropsychology, 13, 1059-1098. 


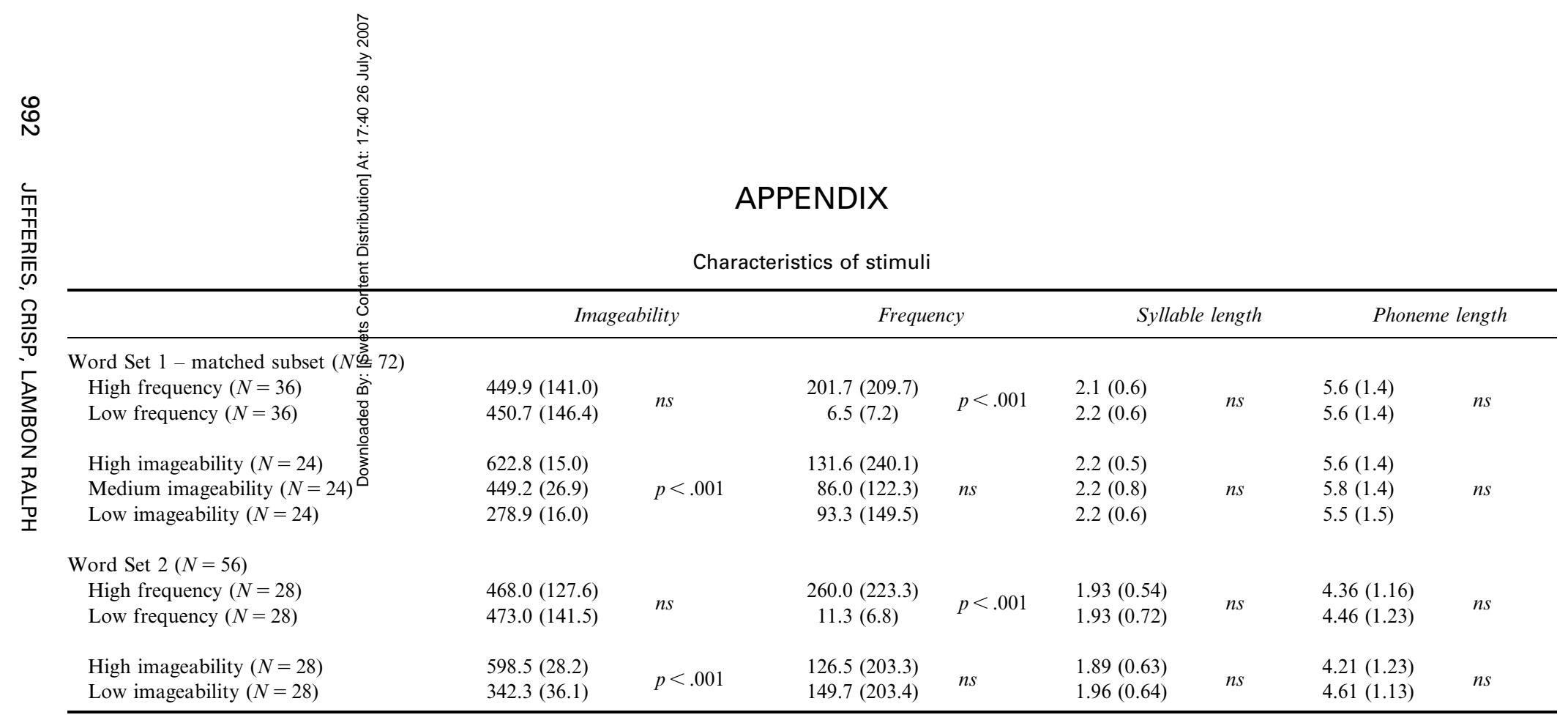

Figures show mean for each group (standard deviations in parentheses). $n s=$ not significant (all $p>.1$ ). Frequency values for Set 1 show Lemma frequency from Celex (Baayen, Piepenbrock, \& van Rijn, 1993). Frequency values for Set 2 are from Kucera and Francis (1967). For Set 2, we were unable to obtain imageability scores for two words and frequency counts for one item. 